\title{
AN INVERSE PROBLEM FOR THE NON-SELF-ADJOINT MATRIX STURM-LIOUVILLE OPERATOR
}

\author{
NATALIA PAVLOVNA BONDARENKO
}

\begin{abstract}
The inverse problem of spectral analysis for the non-self-adjoint matrix SturmLiouville operator on a finite interval is investigated. We study properties of the spectral characteristics for the considered operator, and provide necessary and sufficient conditions for the solvability of the inverse problem. Our approach is based on the constructive solution of the inverse problem by the method of spectral mappings. The characterization of the spectral data in the self-adjoint case is given as a corollary of the main result.
\end{abstract}

\section{Introduction and main results}

Consider the boundary value problem $L=L(Q(x), h, H)$ for the matrix Sturm-Liouville equation

$$
\ell Y:=-Y^{\prime \prime}+Q(x) Y=\lambda Y, \quad x \in(0, \pi),
$$

with the boundary conditions

$$
U(Y):=Y^{\prime}(0)-h Y(0)=0, \quad V(Y):=Y^{\prime}(\pi)+H Y(\pi)=0 .
$$

Here $Y(x)=\left[y_{k}(x)\right]_{k=1, m}$ is a column vector, $\lambda$ is the spectral parameter, and $Q(x)=$ $\left[Q_{j k}(x)\right]_{j, k=1, m}$, where $Q_{j k}(x) \in L_{2}(0, \pi)$ are complex-valued functions. We will subsequently refer to the matrix $Q(x)$ as the potential. The boundary conditions are given by the matrices $h=\left[h_{j k}\right]_{j, k=\overline{1, m}}, H=\left[H_{j k}\right]_{j, k=\overline{1, m}}$, where $h_{j k}$ and $H_{j k}$ are complex numbers.

In this paper, we study the inverse problem of the spectral theory for the matrix SturmLiouville operator $\mathscr{L}, \mathscr{L} Y=\ell Y$, with a domain

$$
\operatorname{Dom}(\mathscr{L})=\left\{Y=\left[y_{k}\right]_{k=1}^{m}: y_{k} \in W_{2}^{2}[0, \pi], k=\overline{1, m}, Y \text { satisfies (2) }\right\} .
$$

Inverse problems consist in recovering differentail operators from their spectral characteristics. Such problems have many applications in science and engineering.

Received January 30, 2018, accepted July 3, 2018.

2010 Mathematics Subject Classification. 34A55 34B24 47E05.

Key words and phrases. Matrix Sturm-Liouville equation, inverse spectral problems, necessary and sufficient conditions, method of spectral mappings. 
Inverse problems for the scalar Sturm-Liouville equation $(m=1)$ have been studied fairly completely (see monographs $[1,2,3,4]$ ). The matrix case is a natural generalization of the scalar one. A significant contribution in the inverse problem theory for the matrix operators was made by Z.S. Agranovich and V.A. Marchenko [5], who studied the matrix Sturm-Liouville operator on the half-line. For inverse problems on the finite interval, uniqueness theorems were proved in works $[6,7,8]$. An algorithm for reconstuction of the matrix potential from the spectral data was presented by V.A. Yurko [9] for the case of the simple spectrum. Then D. Chelkak and E. Korotayev [10] have given the characterization of the spectral data (necessary and sufficient conditions) for the matrix Sturm-Liouville operator with asymptotically simple spectrum, which is a strong restriction. Necessary and sufficient conditions and an algorithm for the solution in the general case, without any restrictions on the behavior of the spectrum, provided in [11]. Ya.V. Mykytyuk and N.S. Trush [12] obtained characterization of the spectral data for the potential from the Sobolev class $W_{2}^{-1}$. Necessary and sufficient conditions for solvability of inverse problems for the matrix Sturm-Liouville operators on the half-line and on the line were provided in [13] and [14], respectively. Inverse problems for first-order systems in the general form were studied in $[15,16,17]$.

All the previous works on the necessary and sufficient conditions for matrix SturmLiouville operators on a finite interval deal with the self-adjoint case: when the matrices $Q$, $h$ and $H$ are Hermitian. In this paper, we study the non-self-adjoint case. We develop the approach of [11], based on the method of spectral mappings [4, 18]. This method allows to reduce an inverse problem to a so-called main equation, which is a linear equation in a suitable Banach space of infinite sequences. The reduction works for non-self-adjoint operators just as well as for self-adjoint ones. Moreover, by necessity one can prove, that the main equation is uniquely solvable. However, by sufficiency it is necessary to require its solvability even in the scalar case (see the example in [4, Section 1.6.3]). For the non-self-adjoint scalar SturmLiouville operator, a constructive solution of the inverse problem by the method of spectral mappings and necessary and sufficient conditions were obtained by S.A. Buterin, C.-T. Shieh and V.A. Yurko [19, 20]. In this paper, we generalize their results, and get necessary and sufficient conditions for the spectral data of the matrix Sturm-Liouville operator.

Proceed to the formulation of the main results. Let $\varphi(x, \lambda)$ and $S(x, \lambda)$ be matrix-solutions of equation (1) under the initial conditions

$$
\varphi(0, \lambda)=I_{m}, \quad \varphi^{\prime}(0, \lambda)=h, \quad S(0, \lambda)=0_{m}, \quad S^{\prime}(0, \lambda)=I_{m}
$$

where $I_{m}$ is the identity $m \times m$ matrix, $0_{m}$ is the zero $m \times m$ matrix. The function $\Delta(\lambda):=$ $\operatorname{det} V(\varphi)$ is called the characteristic function of the boundary value problem $L$. The zeros of the entire function $\Delta(\lambda)$ coincide with the eigenvalues of $L$. 
Let $\omega$ be some $m \times m$ matrix. We will write $L(Q(x), h, H) \in A(\omega)$, if the problem $L$ has a potential from $L_{2}(0, \pi)$ and $h+H+\frac{1}{2} \int_{0}^{\pi} Q(x) d x=\omega$. In the self-adjoint case, the matrix $h+H+\frac{1}{2} \int_{0}^{\pi} Q(x) d x$ is diagonalizable by the unitary transform. In the general case, it is not true, but we restrict ourselves to the class of diagonalizable matrices. Then without loss of generality we can assume that

$$
L \in A(\omega), \quad \omega \in \mathscr{D}=\left\{\omega: \omega=\operatorname{diag}\left\{\omega_{1}, \ldots, \omega_{m}\right\}\right\} .
$$

One can achieve this condition applying the standard transform.

Before we proceed to asymptotics, let us agree to denote by $\left\{\kappa_{n}\right\}$ different sequences from $l_{2}$.

Lemma 1. Let $L \in A(\omega), \omega \in \mathscr{D}$. The boundary value problem $L$ has a countable set of eigenvalues $\left\{\lambda_{n q}\right\}_{n \geq 0, q=\overline{1, m}}$, and

$$
\rho_{n q}:=\sqrt{\lambda_{n q}}=n+\frac{\omega_{q}}{\pi n}+\frac{\kappa_{n}}{n}, \quad q=\overline{1, m} .
$$

Here the eigenvalues are counted with their multiplicities, which they have as zeros of the entire characteristic function $\Delta(\lambda)$.

Since the matrix $\omega$ is diagonal, the proof of Lemma 1 repeats the proof of $[11$, Lemma 1$]$.

Let $\Phi(x, \lambda)=\left[\Phi_{j k}(x, \lambda)\right]_{j, k=\overline{1, m}}$ be a matrix-solution of equation (1) under the boundary conditions $U(\Phi)=I_{m}, V(\Phi)=0_{m}$. We call $\Phi(x, \lambda)$ the Weyl solution for $L$. Put $M(\lambda):=\Phi(0, \lambda)$. The matrix $M(\lambda)=\left[M_{j k}(\lambda)\right]_{j, k=\overline{1, m}}$ is called the Weyl matrix for $L$. The notion of the Weyl matrix is a generalization of the notion of the Weyl function ( $m$-function) for the scalar case (see [1], [4]). The Weyl functions and their generalizations often appear in applications and in pure mathematical problems, and they are natural spectral characteristics in the inverse problem theory for various classes of differential operators.

Using the definition for $\Phi(x, \lambda)$ and $M(\lambda)$, one can easily check that

$$
\begin{aligned}
\Phi(x, \lambda) & =S(x, \lambda)+\varphi(x, \lambda) M(\lambda), \\
M(\lambda) & =-(V(\varphi))^{-1} V(S) .
\end{aligned}
$$

The matrix-function $M(\lambda)$ is meromorphic in $\lambda$ with poles at the eigenvalues $\left\{\lambda_{n q}\right\}$ of $L$. In general, the poles can be multiple, but we put the following restriction.

Assumption 1. All the poles of the matrix-function $M(\lambda)$ are simple.

Note that Assumption 1 corresponds to the case, when the operator does not have associated functions (see [21]). If there is a finite number of multiple poles, one can use the approach of $[19,20]$. 
Define the weight matrices:

$$
\alpha_{n q}:=\operatorname{Res}_{\lambda=\lambda_{n q}} M(\lambda)
$$

Assumption 2. The sequence of the matrices $\left\{\alpha_{n q}\right\}$ is bounded in a matrix norm: $\left\|\alpha_{n q}\right\| \leq C$, for all $n \geq 0, q=\overline{1, m}$.

For definiteness, here and below we consider the following matrix norm

$$
\|A\|=\max _{1 \leq j \leq m} \sum_{k=1}^{m}\left|a_{j k}\right|, \quad A=\left[a_{j k}\right]_{j, k=\overline{1, m}} .
$$

We say that the boundary value problem $L$ belongs to the class $A_{1,2}(\omega)$, if $L \in A(\omega)$ and $L$ satisfies Assumptions 1 and 2.

Note that Assumptions 1 and 2 hold in the following cases.

1. The self-adjoint case (see [11]).

2. Assumption 1 obviously holds, when the eigenvalues are simple. Assumption 2 holds in view of (7), when the eigenvalues are asymptotically simple, i.e. all the values $\omega_{q}$ are distinct.

Let $\left\{\lambda_{n_{k} q_{k}}\right\}_{k \geq 0}$ be all the distinct eigenvalues from the collection $\left\{\lambda_{n q}\right\}_{n \geq 0, q=\overline{1, m}}$. Put

$$
\alpha_{n_{k} q_{k}}^{\prime}:=\alpha_{n_{k} q_{k}}, k \geq 0, \quad \alpha_{n q}^{\prime}=0_{m},(n, q) \notin\left\{\left(n_{k}, q_{k}\right)\right\}_{k \geq 0} .
$$

Fix the numbers $1=m_{1}<m_{2}<\cdots<m_{p}$ so that $\left\{\omega_{m_{s}}\right\}_{s=1}^{p}$ are all the distinct values in the collection $\left\{\omega_{q}\right\}_{q=1}^{m}$. Let $J_{s}=\left\{q: \omega_{q}=\omega_{m_{s}}\right\}$, and $\alpha_{n}^{(s)}=\sum_{q \in J_{s}} \alpha_{n q}^{\prime}, s=\overline{1, p}$. Analogously to $\kappa_{n}$, denote by $\left\{K_{n}\right\}$ different matrix sequences, such that norms of these matrices form sequences from $l_{2}$.

Lemma 2. Let $L \in A_{1,2}(\omega), \omega \in \mathscr{D}$. Then the following relations hold

$$
\begin{gathered}
\alpha_{n}^{(s)}=\frac{2}{\pi} I^{(s)}+K_{n}, \quad s=\overline{1, p}, \quad n \geq 0, \\
\left(I_{m}-I^{(s)}\right) \alpha_{n q}=K_{n}, \quad n \geq 0, s=\overline{1, p}, q \in J_{s},
\end{gathered}
$$

where

$$
I^{(s)}=\left[I_{j k}^{(s)}\right]_{j, k=\overline{1, m}}, \quad I_{j k}^{(s)}=\left\{\begin{array}{l}
1, \quad j=k \in J_{s}, \\
0, \text { otherwise. }
\end{array}\right.
$$

\footnotetext{
${ }^{1}$ In the case of multiple eigenvalues, the same weight matrices $\alpha_{n q}$ occur in $\Lambda$ multiple times. To count each residue in the sum only once, we use the notation $\alpha_{n q}^{\prime}$.
} 
Put $\alpha_{n}:=\sum_{s=1}^{p} \alpha_{n}^{(s)}=\sum_{q=1}^{m} \alpha_{n q}^{\prime}$

Lemma 3. Let $L \in A_{1,2}(\omega), \omega \in \mathscr{D}$. Then the following relation holds

$$
\alpha_{n}=\frac{2}{\pi} I_{m}+\frac{K_{n}}{n}, \quad n \geq 0 .
$$

The data $\Lambda:=\left\{\lambda_{n q}, \alpha_{n q}\right\}_{n \geq 0, q=\overline{1, m}}$ are called the spectral data of the problem $L$. Consider the following inverse problem.

Inverse Problem 1. Given the spectral data $\Lambda$, construct $Q, h$ and $H$.

Let us describe the general strategy of our method. Suppose we know the spectral data $\Lambda$ of some unknown boundary value problem $L \in A_{1,2}(\omega), \omega \in \mathscr{D}$. Choose an arbitrary model boundary value problem $\tilde{L}=L(\tilde{Q}(x), \tilde{h}, \tilde{H}) \in A_{1,2}(\omega)$ (for example, one can take $\tilde{Q}(x)=\frac{2}{\pi} \omega$, $\left.\tilde{h}=0_{m}, \tilde{H}=0_{m}\right)$. We agree that if a certain symbol $\gamma$ denotes an object related to $L$, then the corresponding symbol $\tilde{\gamma}$ with tilde denotes the analogous object related to $\tilde{L}$.

Denote $\lambda_{n q 0}=\lambda_{n q}, \quad \lambda_{n q 1}=\tilde{\lambda}_{n q}, n \geq 0, q=\overline{1, m}$. Let $\psi(x)=\left[\varphi\left(x, \lambda_{n q i}\right)\right]_{n \geq 0, q=\overline{1, m}, i=0,1}$, $\tilde{\psi}(x)=\left[\tilde{\varphi}\left(x, \lambda_{n q i}\right)\right]_{n \geq 0, q=\overline{1, m}, i=0,1}$. It is shown in Section 4, that for each fixed $x \in[0, \pi], \psi(x)$ satisfies the main equation

$$
\tilde{\psi}(x)=\psi(x)(I+\tilde{R}(x))
$$

in a suitable Banach space $B$ of infinite bounded matrix sequences. Here $I$ is the identity operator in $B$, and the operator $\tilde{R}(x)$ is constructed by the model problem $\tilde{L}$ and two collections of spectral data $\Lambda, \tilde{\Lambda}$. Solving the main equation, one can recover the potential $Q$ and the coefficients of the boundary conditions $h$ and $H$ by Algorithm 1, provided in Section 4. Using the main equation, we obtain necessary and sufficient conditions for spectral data of the problem $L$ from $A_{1,2}(\omega)$.

We will write $\left\{\lambda_{n q}, \alpha_{n q}\right\}_{n \geq 0, q=\overline{1, m}} \in \mathrm{Sp}$, if $\lambda_{n q}$ are complex numbers, $\alpha_{n q}$ are $m \times m$ matrices, and for $\lambda_{n q}=\lambda_{k l}$ we always have $\alpha_{n q}=\alpha_{k l}$.

Theorem 1. Let $\omega \in \mathscr{D}$. For data $\left\{\lambda_{n q}, \alpha_{n q}\right\}_{n \geq 0, q=\overline{1, m}} \in S p$ to be the spectral data for a certain problem $L \in A_{1,2}(\omega)$ it is necessary and sufficient to satisfy the following conditions.

(A) The asymptotics (3), (7), (8) (9) are valid, and Assumption 2 holds for $\left\{\alpha_{n q}\right\}$.

(R) The ranks of the matrices $\alpha_{n q}$ coincide with the multiplicities of the corresponding values $\lambda_{n q}{ }^{2}$

(M) The main equation (10) is uniquely solvable.

\footnotetext{
${ }^{2}$ By necessity, the multiplicity of $\lambda_{n q}$ means the number of corresponding linearly independent vector eigenfunctions, equal to the multiplicity of zero of $\Delta(\lambda)$ by Lemma 5 . By sufficiency, the multiplicity means the number of times the value $\lambda_{n q}$ occurs in the given collection.
} 
Condition (M) holds for any choice of a model problem $\tilde{L} \in A_{1,2}(\omega)$ by necessity and for at least one problem $\tilde{L}$ by sufficiency.

Of particular interest are those cases, when the solvability of the main equation can be proved or easily checked, namely, the self-adjoint case, the case of finite perturbations of the spectral data and the case of small perturbations $[19,20]$. As a corollary of Theorem 1 , we derive a result for the self-adjoint case: $Q=Q^{\dagger}, h=h^{\dagger}, H=H^{\dagger}$ (the symbol $\dagger$ stands for the conjugate transpose). Finite perturbations and small perturbations can also be studied analogously to the scalar case. Note that in the self-adjoint case, the problem $L$ always belongs to the class $A_{1,2}(\omega)$ with a diagonalizable $\omega$ (see Section 7 ). Condition (M) can be proved with help of the simplier condition (E), so we obtain the following result.

Theorem 2. Let $\omega=\omega^{\dagger} \in \mathscr{D}$. For data $\left\{\lambda_{n q}, \alpha_{n q}\right\}_{n \geq 0, q=\overline{1, m}} \in S p$ to be the spectral data for a certain self-adjoint problem $L \in A(\omega)$ it is necessary and sufficient to satisfy the following conditions.

(A) The asymptotics (3), (7), (8) (9) are valid.

(R) The ranks of the matrices $\alpha_{n q}$ coincide with the multiplicities of the corresponding values $\lambda_{n q}$.

(S) All $\lambda_{n q}$ are real, $\alpha_{n q}=\left(\alpha_{n q}\right)^{\dagger}, \alpha_{n q} \geq 0$ for all $n \geq 0, q=\overline{1, m}$.

(E) For any row vector $\gamma(\lambda)$ that is entire in $\lambda$, and that satisfy the estimate

$$
\gamma(\lambda)=O(\exp (|\operatorname{Im} \sqrt{\lambda}| \pi)), \quad|\lambda| \rightarrow \infty
$$

if $\gamma\left(\lambda_{n q}\right) \alpha_{n q}=0$ for all $n \geq 0, q=\overline{1, m}$, then $\gamma(\lambda) \equiv 0 .^{3}$

Note that Assumption 2 in (A) is not necessary in the self-adjoint case, because it follows from (9) and the condition $\alpha_{n q} \geq 0$.

As we have already mentioned, the characterization of the spectral data of the self-adjoint matrix Sturm-Liouville operator was obtained earlier in [11]. But the work [11] contains a technical mistake in asymptotics of the weight matrices. In this paper, using the method of [11], we obtain correct necessary and sufficient conditions for the self-adjoint case (Theorem 2) as a corollary of the more general result (Theorem 1).

The paper is organized as follows. At first we study algebraic and analytical properties of the spectral characteristics (Section 2) and prove Lemmas 2 and 3 with asymptotic formulas for the weight matrices (Section 3). In Section 4, we derive the main equation in a suitable Banach space and provide a constructive algorithm for the solution of Inverse Problem 1. We

\footnotetext{
${ }^{3}$ The letters, denoting the conditions, have the following meanings: (A) Asymptotics, (R) Ranks, (M) solvability of the Main equation, (S) Self-adjointness, (E) "Entire function condition". The conditions (C) Completeness, and (PW) Paley-Wiener class condition, appear later (in Section 5).
} 
also prove the unique solvability of the main equation, and this finish the proof of the necessity in Theorem 1. Further, in Section 5, we discuss the connection between the conditions (M), (E), (C) and (PW). Namely, they are connected as follows: $(M) \Rightarrow(E) \Rightarrow(P W) \Leftrightarrow(C)$. Section 6 devoted to the sufficiency in Theorem 1. In Section 7, we collect the results concerning the self-adjoint case, and prove Theorem 2. We also give a reformulation of Theorem 2, using the completeness of some system of vector functions (C).

Notation. Along with $L$ we consider the boundary value problem $L^{*}=L^{*}(Q(x), h, H)$ in the form

$$
\begin{gathered}
\ell^{*} Z:=-Z^{\prime \prime}+Z Q(x)=\lambda Z, \quad x \in(0, \pi), \\
U^{*}(Z):=Z^{\prime}(0)-Z(0) h=0, \quad V^{*}(Z):=Z^{\prime}(\pi)+Z(\pi) H=0,
\end{gathered}
$$

where $Z$ is a row vector. Let $\tilde{L}^{*}=L^{*}(\tilde{Q}(x), \tilde{h}, \tilde{H})$. We agree that if a symbol $\gamma$ denotes an object related to $L$, then $\gamma^{*}$ and $\tilde{\gamma}^{*}$ denote corresponding objects related to $L^{*}$ and $\tilde{L}^{*}$, respectively.

We consider the space of complex column $m$-vectors $\mathbb{C}^{m}$ with the norm

$$
\|Y\|=\max _{1 \leq j \leq m}\left|y_{j}\right|, \quad Y=\left[y_{j}\right]_{j=\overline{1, m}}
$$

the space of complex $m \times m$ matrices $\mathbb{C}^{m \times m}$ with the corresponding induced norm (6), and the space of row vectors $\mathbb{C}^{m, T}$. We use the spaces $L_{2}\left((0, \pi), \mathbb{C}^{m}\right), L_{2}\left((0, \pi), \mathbb{C}^{m, T}\right)$ and $L_{2}\left((0, \pi), \mathbb{C}^{m \times m}\right)$ of column vectors, row vectors and matrices, respectively, with entries from $L_{2}(0, \pi)$. The Hilbert spaces $L_{2}\left((0, \pi), \mathbb{C}^{m}\right)$ and $L_{2}\left((0, \pi), \mathbb{C}^{m, T}\right)$ are equipped with the following scalar products

$$
\begin{aligned}
& (Y, Z)=\int_{0}^{\pi} Y^{\dagger}(x) Z(x) d x=\int_{0}^{\pi} \sum_{j=1}^{m} \bar{y}_{j}(x) z_{j}(x) d x \\
& (Y, Z)=\int_{0}^{\pi} Y(x) Z^{\dagger}(x) d x
\end{aligned}
$$

respectively. Denote $\langle Y, Z\rangle=Y^{\prime} Z-Y Z^{\prime}$.

Put $\rho:=\sqrt{\lambda}, \operatorname{Re} \rho \geq 0, \tau:=\operatorname{Im} \rho$. In estimates and asymptotics, we use the same symbol $C$ for different constants independent of $x, \rho$, etc.

\section{Properties of the spectral data}

The results of this section are valid for any boundary value problem $L$, satisfying Assumption 1. First, we prove an alternative formulation of this assumption.

Lemma 4. Assumption 1 is equivalent to the condition, that all the poles of the matrix function $(V(\varphi(x, \lambda)))^{-1}$ in the $\lambda$-plane are simple. 
Proof. Suppose that $\lambda_{0}$ is a nonsimple pole of $(V(\varphi))^{-1}$, namely

$$
(V(\varphi))^{-1}=\frac{A_{-k}}{\left(\lambda-\lambda_{0}\right)^{k}}+\cdots+\frac{A_{-1}}{\lambda-\lambda_{0}}+A_{0}+\cdots, \quad k>1, \quad A_{k} \neq 0_{m},
$$

in a neighborhood of $\lambda_{0}$. The matrix-function $V(S(x, \lambda))$ is analytical: $V(S(x, \lambda))=V\left(S\left(x, \lambda_{0}\right)\right)$ $+\frac{d}{d \lambda} V\left(S\left(x, \lambda_{0}\right)\right)\left(\lambda-\lambda_{0}\right)+\cdots$. If $\lambda_{0}$ is a simple pole of the Weyl matrix $M(\lambda)$, then $A_{-k} V\left(S\left(x, \lambda_{0}\right)\right)$ $=0_{m}$. The matrix-function $I=(V(\varphi))^{-1} V(\varphi)$ is entire, therefore we also have $A_{-k} V\left(\varphi\left(x, \lambda_{0}\right)\right)=$ $0_{m}$. Since the columns of the matrices $\varphi(x, \lambda)$ and $S(x, \lambda)$ form a fundamental system of solutions of equation (1), every solution $\psi(x, \lambda)$ of this equation can be represented as their linear combination: $\psi(x, \lambda)=\varphi(x, \lambda) A+S(x, \lambda) B$, and it also satisfies the relation $A_{-k} V\left(\psi\left(x, \lambda_{0}\right)\right)=0$. But if we choose the solution $\psi\left(x, \lambda_{0}\right)$, satisfying the initial conditions $\psi\left(\pi, \lambda_{0}\right)=0_{m}, \psi^{\prime}\left(\pi, \lambda_{0}\right)$ $=A_{-k}^{\dagger}$, we get $A_{-k} V\left(\psi\left(x, \lambda_{0}\right)\right) \neq 0$. The contradiction shows, that the simplicity of the poles of $(V(\varphi))^{-1}$ follows from the simplicity of the poles of $M(\lambda)$. The inverse is obvious.

Lemma 5. The zeros of the characteristic function $\Delta(\lambda)$ coincide with the eigenvalues of the boundary value problem $L$. The multiplicity of each zero $\lambda_{0}$ of the function $\Delta(\lambda)$ equals to the multiplicity of the corresponding eigenvalue (by the multiplicity of the eigenvalue we mean the number of the corresponding linearly independent vector eigenfunctions).

Proof. 1. Let $\lambda_{0}$ be an eigenvalue of $L$, and let $Y^{0}$ be an eigenfunction corresponding to $\lambda_{0}$. Let us show that $Y^{0}(x)=\varphi\left(x, \lambda_{0}\right) Y^{0}(0)$. Clearly, $Y^{0}(0)=\varphi(0, \lambda) Y^{0}(0)$. It follows from $U\left(Y^{0}\right)=0$ that $Y^{0^{\prime}}(0)=h Y^{0}(0)=\varphi(0, \lambda) Y^{0}(0)$. Thus, $Y^{0}(x)$ and $\varphi\left(x, \lambda_{0}\right) Y^{0}(0)$ are the solutions for the same initial value problem for equation (1). Consequently, they are equal.

2. Let us have exactly $k$ linearly independent eigenfunctions $Y^{1}, Y^{2}, \ldots, Y^{k}$ corresponding to the eigenvalue $\lambda_{0}$. Choose the invertible $m \times m$ matrix $C$ such that the first $k$ columns of $\varphi\left(x, \lambda_{0}\right) C$ coincide with the eigenfunctions. Consider $Y(x, \lambda):=\varphi(x, \lambda) C$, $Y(x, \lambda)=\left[Y_{q}(x, \lambda)\right]_{q=\overline{1, m}}, Y_{q}\left(x, \lambda_{0}\right)=Y^{q}(x), q=\overline{1, k}$. Clearly, the zeros of $\Delta_{1}(\lambda):=\operatorname{det} V(Y)=$ $\operatorname{det} V(\varphi) \cdot \operatorname{det} C$ coincide with the zeros of $\Delta(\lambda)$ counting with their multiplicities. Note that $\lambda=\lambda_{0}$ is a zero of each of the columns $V\left(Y_{1}\right), \ldots, V\left(Y_{k}\right)$. Hence, if $\lambda_{0}$ is the zero of the determinants $\Delta_{1}(\lambda)$ and $\Delta(\lambda)$ with the multiplicity $p$, then $p \geq k$.

3. Suppose that $p>k$. Rewrite $\Delta_{1}(\lambda)$ in the form

$$
\begin{gathered}
\Delta_{1}(\lambda)=\left(\lambda-\lambda_{0}\right)^{k} \Delta_{2}(\lambda), \\
\Delta_{2}(\lambda)=\operatorname{det}\left[\frac{V\left(Y_{1}\right)}{\lambda-\lambda_{0}}, \ldots, \frac{V\left(Y_{k}\right)}{\lambda-\lambda_{0}}, V\left(Y_{k+1}\right), \ldots, V\left(Y_{m}\right)\right] .
\end{gathered}
$$

In view of our supposition, we have $\Delta_{2}\left(\lambda_{0}\right)=0$, i. e. there exist not all zero coefficients $\alpha_{q}$, $q=\overline{1, m}$ such that

$$
\sum_{q=1}^{k} \alpha_{q} \frac{d V\left(Y_{q}\left(x, \lambda_{0}\right)\right)}{d \lambda}+\sum_{q=k+1}^{m} \alpha_{q} V\left(Y_{q}\left(x, \lambda_{0}\right)\right)=0
$$


If $\alpha_{q}=0$ for $q=\overline{1, k}$, then the function

$$
Y^{+}(x, \lambda):=\sum_{q=k+1}^{m} \alpha_{q} Y_{q}(x, \lambda)
$$

for $\lambda=\lambda_{0}$ is an eigenfunction, corresponding to $\lambda_{0}$, that is linearly independent with $Y^{q}$, $q=\overline{1, k}$. Since the eigenvalue $\lambda_{0}$ has exactly $k$ corresponding eigenfunctions, we arrive at a contradiction.

Otherwise we consider the function

$$
Y^{+}(x, \lambda):=\sum_{q=1}^{k} \alpha_{q} Y_{q}(x, \lambda)+\left(\lambda-\lambda_{0}\right) \sum_{q=k+1}^{m} \alpha_{q} Y_{q}(x, \lambda) .
$$

Now we plan to use the simplicity of the poles of $(V(\varphi))^{-1}$, following from Assumption 1 by Lemma 4. Recall the following well-known fact (see [5, Lemma 2.2.1]):

The inverse $(V(\varphi))^{-1}$ has a simple pole at $\lambda=\lambda_{0}$ if and only if the relations at $\lambda=\lambda_{0}$ :

$$
\begin{gathered}
V(\varphi) a=0, \\
\frac{d}{d \lambda} V(\varphi) a+V(\varphi) b=0,
\end{gathered}
$$

where $a$ and $b$ are constant vectors, yield $a=0$.

The function $Y^{+}$has the form $Y^{+}(x, \lambda)=V(\varphi) a+\left(\lambda-\lambda_{0}\right) V(\varphi) b, a \neq 0$. In view of (12), the relations (13) are satisfied, and we arrive at a contradiction with Assumption 1. Thus, $\Delta_{2}\left(\lambda_{0}\right) \neq 0$ and $p=k$.

Lemma 6. The ranks of the residue-matrices of the Weyl matrix $M(\lambda)$ coincide with the multiplicities of the corresponding eigenvalues of $L$.

Under Assumption 1, the proof of Lemma 6 does not differ from the proof in the selfadjoint case (see [11, Lemma 4]).

Now let us consider the problem $L^{*}$, defined by (11). It is easy to check that

$$
\langle Z, Y\rangle_{x=0}=U^{*}(Z) Y(0)-Z(0) U(Y), \quad\langle Z, Y\rangle_{x=\pi}=V^{*}(Z) Y(\pi)-Z(\pi) V(Y) .
$$

where $\langle Z, Y\rangle=Z^{\prime} Y-Z Y^{\prime}$. If $Y(x, \lambda)$ and $Z(x, \lambda)$ satisfy the equations $\ell Y(x, \lambda)=\lambda Y(x, \lambda)$, $\ell^{*} Z(x, \mu)=\mu Z(x, \mu)$, respectively, then

$$
\frac{d}{d x}\langle Z(x, \mu), Y(x, \lambda)\rangle=(\lambda-\mu) Z(x, \mu) Y(x, \lambda),
$$

Introduce the matrices $\varphi^{*}(x, \lambda), S^{*}(x, \lambda)$ and $\Phi^{*}(x, \lambda)$, satisfying the equation $\ell^{*} Z=\lambda Z$ and the conditions

$$
\varphi^{*}(0, \lambda)=S^{* \prime}(0, \lambda)=U^{*}\left(\Phi^{*}\right)=I_{m}, \quad \varphi^{* \prime}(0, \lambda)=h, \quad S^{*}(0, \lambda)=V^{*}\left(\Phi^{*}\right)=0_{m} .
$$


Denote $M^{*}(\lambda):=\Phi^{*}(0, \lambda)$.

In view of (15), the expression $\left\langle\Phi^{*}(x, \lambda), \Phi(x, \lambda)\right\rangle$ does not depend on $x$. Using (14), we obtain

$$
\left\langle\Phi^{*}(x, \lambda), \Phi(x, \lambda)\right\rangle_{x=0}=M(\lambda)-M^{*}(\lambda), \quad\left\langle\Phi^{*}(x, \lambda), \Phi(x, \lambda)\right\rangle_{x=\pi}=0_{m} .
$$

Hence

$$
M(\lambda) \equiv M^{*}(\lambda)
$$

and consequently, the spectral data of the problems $L$ and $L^{*}$ coincide.

Lemma 7. Let $\lambda_{0}, \lambda_{1}$ be eigenvalues of $L, \lambda_{0} \neq \lambda_{1}$, and $\alpha_{i}=\underset{\lambda=\lambda_{i}}{\operatorname{Res}} M(\lambda), i=0,1$. The following relations hold

$$
\begin{aligned}
& \alpha_{0} \int_{0}^{\pi} \varphi^{*}\left(x, \lambda_{0}\right) \varphi\left(x, \lambda_{0}\right) d x \alpha_{0}=\alpha_{0}, \\
& \alpha_{0} \int_{0}^{\pi} \varphi^{*}\left(x, \lambda_{0}\right) \varphi\left(x, \lambda_{1}\right) d x \alpha_{1}=0_{m} .
\end{aligned}
$$

Proof. Using (14) and (15), we derive

$$
\begin{aligned}
& \int_{0}^{\pi} \varphi^{*}\left(x, \lambda_{0}\right) \varphi\left(x, \lambda_{0}\right) d x=\lim _{\lambda \rightarrow \lambda_{0}} \frac{\left.\left\langle\varphi^{*}\left(x, \lambda_{0}\right), \varphi(x, \lambda)\right\rangle\right|_{0} ^{\pi}}{\lambda-\lambda_{0}} \\
& =\lim _{\lambda \rightarrow \lambda_{0}} \frac{V^{*}\left(\varphi^{*}\left(x, \lambda_{0}\right)\right) \varphi(\pi, \lambda)-\varphi^{*}\left(\pi, \lambda_{0}\right) V(\varphi(x, \lambda))}{\lambda-\lambda_{0}} .
\end{aligned}
$$

In view of (5), the product $V(\varphi) M(\lambda)$ is an entire function of $\lambda$. Taking its residues at $\lambda_{0}$, we get

$$
V\left(\varphi\left(x, \lambda_{0}\right)\right) \alpha_{0}=0_{m}
$$

Similarly $\alpha_{0} V^{*}\left(\varphi^{*}\left(x, \lambda_{0}\right)\right)=0_{m}$. Consequently, we calculate

$$
\begin{aligned}
& \alpha_{0} \int_{0}^{\pi} \varphi^{*}\left(x, \lambda_{0}\right) \varphi\left(x, \lambda_{0}\right) d x \alpha_{0} \\
& \quad=\alpha_{0} \varphi^{*}\left(\pi, \lambda_{0}\right) \lim _{\lambda \rightarrow \lambda_{0}} \frac{V(\varphi(x, \lambda))}{\lambda-\lambda_{0}} \times \lim _{\lambda \rightarrow \lambda_{0}}\left(\lambda-\lambda_{0}\right)(V(\varphi(x, \lambda)))^{-1} V(S(x, \lambda)) \\
& \quad=\alpha_{0} \varphi^{*}\left(\pi, \lambda_{0}\right) V\left(S\left(x, \lambda_{0}\right)\right)=-\alpha_{0}\left\langle\varphi^{*}\left(x, \lambda_{0}\right), S\left(x, \lambda_{0}\right)\right\rangle_{x=\pi} \\
& \quad=-\alpha_{0}\left\langle\varphi^{*}\left(x, \lambda_{0}\right), S\left(x, \lambda_{0}\right)\right\rangle_{x=0}=\alpha_{0}
\end{aligned}
$$

Similarly one can derive the second relation of the lemma.

Lemma 8. Let $\left\{\lambda_{n q}, \alpha_{n q}\right\}_{n \geq 0, q=1, m}$ be the spectral data of the problem L, satisfying Assumptions 1 and 2. Then (E) is valid. 
Proof. Let $\gamma(\lambda)$ be a function described in (E). In view of (17), we have

$$
V\left(\varphi\left(x, \lambda_{n q}\right)\right) \alpha_{n q}=0_{m}, \quad n \geq 0, q=\overline{1, m} .
$$

Since

$$
\operatorname{rank} V\left(\varphi\left(x, \lambda_{n q}\right)\right)+\operatorname{rank} \alpha_{n q}=m
$$

and $\gamma\left(\lambda_{n q}\right) \alpha_{n q}=0$, we get $\gamma\left(\lambda_{n q}\right)=C_{n q} V\left(\varphi\left(x, \lambda_{n q}\right)\right)$, i. e. the row $\gamma\left(\lambda_{n q}\right)$ is a linear combination of the rows of the matrix $V\left(\varphi\left(x, \lambda_{n q}\right)\right)$ (here $C_{n q}$ is a row of coefficients). Consider

$$
f(\lambda)=\gamma(\lambda)(V(\varphi(x, \lambda)))^{-1}
$$

The matrix-function $(V(\varphi(x, \lambda)))^{-1}$ has simple poles in $\lambda=\lambda_{n q}$, therefore, we calculate

$$
\begin{aligned}
\operatorname{Res}_{\lambda=\lambda_{n q}} f(\lambda) & =\gamma\left(\lambda_{n q}\right) \operatorname{Res}_{\lambda=\lambda_{n q}}(V(\varphi(x, \lambda)))^{-1} \\
& =C_{n q} \lim _{\lambda \rightarrow \lambda_{n q}} V(\varphi(x, \lambda)) \lim _{\lambda \rightarrow \lambda_{n q}}\left(\lambda-\lambda_{n q}\right)(V(\varphi(x, \lambda)))^{-1}=0 .
\end{aligned}
$$

Hence, $f(\lambda)$ is entire. It is easy to show that

$$
\left\|(V(\varphi(x, \lambda)))^{-1}\right\| \leq C_{\delta}|\rho|^{-1} \exp (-|\tau| \pi), \quad \rho \in G_{\delta},
$$

where $G_{\delta}=\left\{\rho:|\rho-k| \geq \delta, k=0,1,2, \ldots,|\rho| \geq \rho^{*}\right\}, \delta>0$. From this we conclude that $\|f(\lambda)\| \leq$ $\frac{C}{|\rho|}$ in $G_{\delta}$. By the maximum principle this estimate is valid in the whole $\lambda$-plane. Using Liouville's theorem, we obtain $f(\lambda) \equiv 0$. Consequently, $\gamma(\lambda) \equiv 0$.

\section{Asymptotics}

In this section, we prove Lemmas 2 and 3, providing asymptotic formulas for the weight matrices $\alpha_{n q}$.

Lemma 9. For $|\rho| \rightarrow \infty$, the following asymptotic formulae hold

$$
\begin{aligned}
V(\varphi) & =-\rho \sin \rho \pi \cdot I_{m}+\omega \cos \rho \pi+\kappa(\rho), \\
\kappa(\rho) & =\frac{1}{2} \int_{0}^{\pi} Q(t) \cos \rho(\pi-2 t) d t+O\left(\frac{\exp (|\tau| \pi)}{\rho}\right) ; \\
V(S) & =\cos \rho \pi \cdot I_{m}+\frac{\sin \rho \pi}{\rho} \omega_{0}+\frac{\kappa_{0}(\rho)}{\rho}, \quad \omega_{0}=H+\frac{1}{2} \int_{0}^{\pi} Q(t) d t \\
\kappa_{0}(\rho) & =-\frac{1}{2} \int_{0}^{\pi} \sin \rho(\pi-2 t) Q(t) d t+O\left(\frac{\exp (|\tau| \pi)}{\rho}\right) .
\end{aligned}
$$


Proof. The assertion of the lemma immediately follows from the standard asymptotics:

$$
\begin{aligned}
\varphi(x, \lambda) & =\cos \rho x \cdot I_{m}+\frac{\sin \rho x}{\rho} Q_{1}(x)+\frac{1}{2 \rho} \int_{0}^{x} \sin \rho(x-2 t) Q(t) d t+O\left(\frac{\exp (|\tau| x)}{\rho^{2}}\right), \\
\varphi^{\prime}(x, \lambda) & =-\rho \sin \rho x \cdot I_{m}+\cos \rho x Q_{1}(x)+\frac{1}{2} \int_{0}^{x} \cos \rho(x-2 t) Q(t) d t+O\left(\frac{\exp (|\tau| x)}{\rho}\right),
\end{aligned}
$$

where $Q_{1}(x)=h+\frac{1}{2} \int_{0}^{x} Q(t) d t$, and

$$
\begin{aligned}
& S(x, \lambda)=\frac{\sin \rho x}{\rho} \cdot I_{m}-\frac{\cos \rho x}{2 \rho^{2}} \int_{0}^{x} Q(t) d t+\frac{1}{2 \rho^{2}} \int_{0}^{x} \cos \rho(x-2 t) Q(t) d t+O\left(\frac{\exp (|\tau| x)}{\rho^{3}}\right), \\
& S^{\prime}(x, \lambda)=\cos \rho x \cdot I_{m}+\frac{\sin \rho x}{2 \rho} \int_{0}^{x} Q(t) d t-\frac{1}{2 \rho} \int_{0}^{x} \sin \rho(x-2 t) Q(t) d t+O\left(\frac{\exp (|\tau| x)}{\rho^{2}}\right) .
\end{aligned}
$$

These formulas can be derived similarly to the scalar ones from [4, Lemma 1.1.2, Theorem 1.1.3].

Proof of Lemma 2. Consider the contour

$$
\gamma_{n}^{(s)}:=\left\{\lambda: \lambda=n^{2}+\frac{2}{\pi} \mu,\left|\mu-\omega_{m_{s}}\right|=R:=\frac{1}{2} \min _{j, k}\left|\omega_{j}-\omega_{k}\right|\right\} .
$$

Then for sufficiently large $n$, by virtue of (3) and the residue theorem,

$$
\alpha_{n}^{(s)}=\frac{1}{2 \pi i} \int_{\gamma_{n}^{(s)}} M(\lambda) d \lambda .
$$

Further in this proof, we fix $s=\overline{1, p}$ and a sufficiently large $n$, and consider only $\lambda=$ $n^{2}+\frac{2}{\pi} \mu \in \gamma_{n}^{(s)}$. Taking a square root, we get

$$
\rho=\sqrt{\lambda}=n+\frac{\mu}{\pi n}+\frac{\kappa_{n}(\mu)}{n} .
$$

Here and below $\left\{\kappa_{n}(\mu)\right\}$ denotes different sequences, depending on $\mu$, but is majorized by a constant sequence from $l_{2}$, independent of $\mu$ :

$$
\forall \mu:\left|\mu-\omega_{m_{s}}\right|=R, \quad\left|\kappa_{n}(\mu)\right| \leq \kappa_{n}, \quad \sum_{n} \kappa_{n}^{2}<\infty
$$

Similarly, $\left\{K_{n}(\mu)\right\}$ denotes sequences of matrices, whose norms form scalar sequences $\left\{\kappa_{n}(\mu)\right\}$.

It follows from (19) and (20), that for $\lambda \in \gamma_{n}^{(s)}$,

$$
V(\varphi)=(-1)^{n}\left(-\mu I_{m}+\omega+K_{n}(\mu)\right), \quad V(S)=(-1)^{n}\left(I_{m}+\frac{K_{n}(\mu)}{n}\right) .
$$

Substitute this into (5):

$$
M(\lambda)=\left(\mu I_{m}-\omega+K_{n}(\mu)\right)^{-1}\left(I_{m}+\frac{K_{n}(\mu)}{n}\right) .
$$


Since $\left|\mu-\omega_{q}\right| \geq R$ for all $q=\overline{1, m}$, the inverse $M_{0}(\mu):=\left(\mu I_{m}-\omega\right)^{-1}$ is bounded, and

$$
M(\lambda)-M_{0}(\mu)=K_{n}(\mu), \quad \frac{1}{2 \pi i} \int_{\gamma_{n}^{(s)}}\left(M(\lambda)-M_{0}(\mu)\right) d \lambda=K_{n} .
$$

We calculate

$$
\frac{1}{2 \pi i} \int_{\gamma_{n}^{(s)}} M_{0}(\mu) d \lambda=\frac{2}{\pi} \cdot \frac{1}{2 \pi i} \int_{\left|\mu-\omega_{m_{s}}\right|=R}\left(\mu I_{m}-\omega\right)^{-1} d \mu=\frac{2}{\pi} I^{(s)} .
$$

Together with (23), this gives (7).

By (3) and (19), $V\left(\varphi\left(x, \lambda_{n q}\right)\right)=(-1)^{n}\left(\omega-\omega_{q} I_{m}+K_{n}\right)$. By Assumption $2,\left\|\alpha_{n q}\right\| \leq C$. Using these facts together with (18), we obtain $\left(\omega-\omega_{q} I_{m}\right) \alpha_{n q}=K_{n}$. This relation yields (8).

Lemma 10. Let a matrix $A$ be such that $\|A\|<R$. Then

$$
\frac{1}{2 \pi i} \int_{|\mu|=R}\left(\mu I_{m}-A\right)^{-1} d \mu=I_{m} .
$$

Proof. The matrix-function $F(\mu)=\left(\mu I_{m}-A\right)^{-1}$ is analytic outside the circle $|\mu|<R$. Therefore,

$$
\frac{1}{2 \pi i} \int_{|\mu|=R} F(\mu) d \mu=-\operatorname{Res}_{\mu=\infty} F(\mu) .
$$

The Laurent series

$$
F(\mu)=\frac{1}{\mu}\left(I_{m}+\frac{A}{\mu}+\frac{A^{2}}{\mu^{2}}+\cdots\right)
$$

converge uniformly when $|\mu| \geq R>\|A\|$. Therefore

$$
\underset{\mu=\infty}{\operatorname{Res}} F(\mu)=-I_{m}
$$

that yields the assertion of the lemma.

Proof of Lemma 3. Note that in fact, the asymptotics

$$
\alpha_{n}=\frac{2}{\pi} I_{m}+K_{n}, \quad n \geq 0,
$$

are already proved. In order to improve the estimate, we will work with the remainder $\kappa(\rho)$ in (19).

Substituting the representation

$$
\begin{aligned}
\varphi^{\prime}(x, \lambda)= & -\rho \sin \rho x \cdot I_{m}+\cos \rho x Q_{1}(x)+\frac{1}{2} \int_{0}^{x} \cos \rho(x-2 t) Q(t) d t \\
& +\frac{\sin \rho x}{2 \rho} \int_{0}^{x} Q(t) Q_{1}(t) d t-\frac{1}{2 \rho} \int_{0}^{x} \sin \rho(x-2 t) Q(t) Q_{1}(t) d t \\
& +\frac{1}{2 \rho} \int_{0}^{x} \cos \rho(x-t) Q(t) \int_{0}^{t} \sin \rho(t-2 s) Q(s) d s d t+O\left(\frac{\exp (|\tau| x)}{\rho^{2}}\right),
\end{aligned}
$$




$$
Q_{1}(x):=h+\frac{1}{2} \int_{0}^{x} Q(t) d t
$$

and (21) into $V(\varphi)=\varphi^{\prime}(\pi, \lambda)+H \varphi(\pi, \lambda)$, we arrive at (19) with

$$
\begin{aligned}
\kappa(\rho)= & \frac{1}{2} \int_{0}^{\pi} \cos \rho(\pi-2 t) Q(t) d t+\frac{\sin \rho \pi}{\rho}\left(\frac{1}{2} \int_{0}^{\pi} Q(t) Q_{1}(t) d t+H Q_{1}(\pi)\right) \\
& +\frac{1}{2 \rho} \int_{0}^{\pi} \sin \rho(\pi-2 t)\left(H Q(t)-Q(t) Q_{1}(t)\right) d t \\
& +\frac{1}{2 \rho} \int_{0}^{\pi} \cos \rho(\pi-t) Q(t) \int_{0}^{t} \sin \rho(t-2 s) Q(s) d s d t+O\left(\frac{\exp (|\tau| \pi)}{\rho^{2}}\right) .
\end{aligned}
$$

Consider the contour

$$
\gamma_{n}:=\left\{\lambda: \lambda=n^{2}+\frac{2}{\pi} \mu,|\mu|=3\|\omega\|\right\} .
$$

Further in this proof, we fix a sufficiently large $n$, such that

$$
\alpha_{n}=\frac{1}{2 \pi i} \int_{\gamma_{n}} M(\lambda) d \lambda
$$

and consider only $\lambda=n^{2}+\frac{2}{\pi} \mu \in \gamma_{n}$. Then the square root of $\lambda$ takes the form (24).

Substitute (24) into (25). Then the first integral in (25) equals

$$
\frac{1}{2} \int_{0}^{\pi} \cos n(\pi-2 t) Q(t) d t+\frac{K_{n}(\mu)}{n},
$$

and all the other terms are $\frac{K_{n}(\mu)}{n}$. Then by (5), (19), (20), we get

$$
M(\lambda)=\left(\mu I_{m}-\omega+L_{n}+\frac{K_{n}(\mu)}{n}\right)^{-1}\left(I_{m}+\frac{K_{n}(\mu)}{n}\right), \quad \lambda \in \gamma_{n},
$$

where $L_{n}$ is a matrix sequence independent of $\mu$, and $\left\{\left\|L_{n}\right\|\right\} \in l_{2}$. Thus, for large $n,\left\|L_{n}\right\|+$ $\left\|K_{n}(\mu) / n\right\| \leq\|\omega\|$ and the inverses $\left(\mu I_{m}-\omega+L_{n}+\frac{K_{n}(\mu)}{n}\right)^{-1}$ and $\left(\mu I_{m}-\omega+L_{n}\right)^{-1}$ are bounded for $|\mu|=3\|\omega\|$. Therefore

$$
\frac{1}{2 \pi i} \int_{|\mu|=3\|\omega\|} M(\lambda) d \mu=\frac{1}{2 \pi i} \int_{|\mu|=3\|\omega\|}\left(\mu I_{m}-\omega+L_{n}\right)^{-1} d \mu+\frac{K_{n}}{n} .
$$

Applying Lemma 10 to the right-hand side and changing $d \mu$ to $d \lambda$, we arrive at (9).

\section{Solution of Inverse Problem 1}

Let the spectral data $\Lambda$ of the boundary value problem $L \in A_{1,2}(\omega), \omega \in \mathscr{D}$, be given. 
Denote

$$
D(x, \lambda, \mu)=\frac{\left\langle\varphi^{*}(x, \mu), \varphi(x, \lambda)\right\rangle}{\lambda-\mu}=\int_{0}^{x} \varphi^{*}(t, \mu) \varphi(t, \lambda) d t .
$$

We choose an arbitrary model boundary value problem $\tilde{L}=L(\tilde{Q}(x), \tilde{h}, \tilde{H}) \in A_{1,2}(\omega)$ (for example, one can take $\left.\tilde{Q}(x)=\frac{2}{\pi} \omega, \tilde{h}=0_{m}, \tilde{H}=0_{m}\right)$. Note that for this choice of the model problem $\omega=\tilde{\omega}$, therefore the eigenvalues $\tilde{\lambda}_{n q}$ and the weight matrices $\tilde{\alpha}_{n q}$ of $\tilde{L}$ satisfy the same asymptotic formulae (3), (7), (8) and (9), as $\lambda_{n q}$ and $\alpha_{n q}$. Put

$\xi_{n}=\sum_{q=1}^{m}\left|\rho_{n q}-\tilde{\rho}_{n q}\right|+\sum_{s=1}^{p} \sum_{q \in J_{s}}\left|\rho_{n q}-\rho_{n m_{s}}\right|+\sum_{s=1}^{p} \sum_{q \in J_{s}}\left|\tilde{\rho}_{n q}-\tilde{\rho}_{n m_{s}}\right|+\sum_{s=1}^{p} \frac{1}{n}\left\|\alpha_{n}^{(s)}-\tilde{\alpha}_{n}^{(s)}\right\|+\left\|\alpha_{n}-\tilde{\alpha}_{n}\right\|$,

then

$$
\Omega:=\left(\sum_{n=0}^{\infty}\left((n+1) \xi_{n}\right)^{2}\right)^{1 / 2}<\infty, \quad \sum_{n=0}^{\infty} \xi_{n}<\infty
$$

Denote

$$
\begin{gathered}
\lambda_{n q 0}=\lambda_{n q}, \quad \lambda_{n q 1}=\tilde{\lambda}_{n q}, \quad \rho_{n q 0}=\rho_{n q}, \quad \rho_{n q 1}=\tilde{\rho}_{n q}, \quad \alpha_{n q 0}^{\prime}=\alpha_{n q}^{\prime}, \quad \alpha_{n q 1}^{\prime}=\tilde{\alpha}_{n q}^{\prime}, \\
\varphi_{n q i}(x)=\varphi\left(x, \lambda_{n q i}\right), \quad \tilde{\varphi}_{n q i}(x)=\tilde{\varphi}\left(x, \lambda_{n q i}\right), \quad \varphi_{n q i}^{*}(x)=\varphi^{*}\left(x, \lambda_{n q i}\right), \quad \tilde{\varphi}_{n q i}^{*}(x)=\tilde{\varphi}^{*}\left(x, \lambda_{n q i}\right), \\
n \geq 0, \quad q=\overline{1, m}, \quad i=0,1 .
\end{gathered}
$$

By the standard way (see [4, Lemma 1.6.2]), using Schwarz's lemma, we get

Lemma 11. The following estimates are valid for $x \in[0, \pi], n, k \geq 0, q, l, r=\overline{1, m}, i, j, s=0,1$ :

$$
\begin{gathered}
\left\|\varphi_{n q i}(x)\right\| \leq C, \quad\left\|\varphi_{n q i}(x)-\varphi_{n l j}(x)\right\| \leq C\left|\rho_{n q i}-\rho_{n l j}\right|, \\
\left\|D\left(x, \lambda_{n q i}, \lambda_{k l j}\right)\right\| \leq \frac{C}{|n-k|+1}, \quad\left\|D\left(x, \lambda_{n q i}, \lambda_{k l j}\right)-D\left(x, \lambda_{n q i}, \lambda_{k r s}\right)\right\| \leq \frac{C\left|\rho_{k l j}-\rho_{k r s}\right|}{|n-k|+1} .
\end{gathered}
$$

The analogous estimates are also valid for $\tilde{\varphi}_{n q i}(x), \tilde{D}\left(x, \lambda_{n q i}, \lambda_{k l j}\right)$, as well as for similar matrix functions, related to the problems $L^{*}, \tilde{L}^{*}$.

The next theorem plays a crucial role in the derivation of the main equation of the inverse problem. It can be proved similarly to [9, Lemma 1] by the contour integral method.

Theorem 3. The following relations hold

$$
\begin{gathered}
\tilde{\varphi}(x, \lambda)=\varphi(x, \lambda)+\sum_{k=0}^{\infty} \sum_{l=1}^{m}\left(\varphi_{k l 0}(x) \alpha_{k l 0}^{\prime} \tilde{D}\left(x, \lambda, \lambda_{k l 0}\right)-\varphi_{k l 1}(x) \alpha_{k l 1}^{\prime} \tilde{D}\left(x, \lambda, \lambda_{k l 1}\right)\right) \\
\tilde{D}(x, \lambda, \mu)-D(x, \lambda, \mu)=\sum_{k=0}^{\infty} \sum_{l=1}^{m}\left(D\left(x, \lambda_{k l 0}, \mu\right) \alpha_{k l 0}^{\prime} \tilde{D}\left(x, \lambda, \lambda_{k l 0}\right)-D\left(x, \lambda_{k l 1}, \mu\right) \alpha_{k l 1}^{\prime} \tilde{D}\left(x, \lambda, \lambda_{k l 1}\right)\right) .
\end{gathered}
$$

Both series converge absolutely and uniformly with respect to $x \in[0, \pi]$ and $\lambda, \mu$ on compact sets. 
Analogously one can obtain the following relation

$$
\tilde{\Phi}(x, \lambda)=\Phi(x, \lambda)+\sum_{k=0}^{\infty} \sum_{l=1}^{m} \sum_{j=0}^{1}(-1)^{j} \varphi_{k l j}(x) \alpha_{k l j}^{\prime} \frac{\left\langle\tilde{\varphi}_{k l j}^{*}(x), \tilde{\Phi}(x, \lambda)\right\rangle}{\lambda-\lambda_{k l j}} .
$$

It follows from Theorem 3 that

$$
\begin{array}{r}
\tilde{\varphi}_{n q i}(x)=\varphi_{n q i}(x)+\sum_{k=0}^{\infty} \sum_{l=1}^{m}\left(\varphi_{k l 0}(x) \alpha_{k l 0}^{\prime} \tilde{D}\left(x, \lambda_{n q i}, \lambda_{k l 0}\right)-\varphi_{k l 1}(x) \alpha_{k l 1}^{\prime} \tilde{D}\left(x, \lambda_{n q i}, \lambda_{k l 1}\right)\right), \\
\alpha_{\eta r j}^{\prime} \tilde{D}\left(x, \lambda_{n q i}, \lambda_{\eta r j}\right)-\alpha_{\eta r j}^{\prime} D\left(x, \lambda_{n q i}, \lambda_{\eta r j}\right)= \\
\sum_{k=0}^{\infty} \sum_{l=1}^{m}\left(\alpha_{\eta r j}^{\prime} D\left(x, \lambda_{k l 0}, \lambda_{\eta r j}\right) \alpha_{k l 0}^{\prime} \tilde{D}\left(x, \lambda_{n q i}, \lambda_{k l 0}\right)\right. \\
\left.-\alpha_{\eta r j}^{\prime} D\left(x, \lambda_{k l 1}, \lambda_{\eta r j}\right) \alpha_{k l 1}^{\prime} \tilde{D}\left(x, \lambda_{n q i}, \lambda_{k l 1}\right)\right) .(33)
\end{array}
$$

for $n, \eta \geq 0, q, r=\overline{1, m}, i, j=0,1$.

For each fixed $x \in[0, \pi]$, the relation (32) can be considered as a system of linear equations with respect to $\varphi_{n q i}(x), n \geq 0, q=\overline{1, m}, i=0,1$. But the series in (32) converges only "with brackets". Therefore, it is not convenient to use (32) as a main equation of the inverse problem. Below we will transfer (32) to a linear equation in a corresponding Banach space of sequences.

Introduce collections $G_{n}=\left\{\rho_{n q i}\right\}_{q=\overline{1, m}, i=0,1}, n \geq 0$. Fix $n$ and, for convenience, renumerate the elements of the collection: $G_{n}=\left\{g_{i}\right\}_{i=1}^{2 m}$. Consider a finite-dimensional space $B\left(G_{n}\right)=\left\{f: G_{n} \rightarrow \mathbb{C}^{m \times m}\right\}$ of matrix-functions $f$, such that $f\left(g_{i}\right)=f\left(g_{j}\right)$ if $g_{i}=g_{j}$, with the norm

$$
\|f\|_{B\left(G_{n}\right)}=\max \left\{\max _{i}\left\|f\left(g_{i}\right)\right\|, \max _{i, j: g_{i} \neq g_{j}}\left\|f\left(g_{i}\right)-f\left(g_{j}\right)\right\| \cdot\left|g_{i}-g_{j}\right|^{-1}\right\} .
$$

Introduce a Banach space of infinite row vectors

$$
B=\left\{f=\left\{f_{n}\right\}_{n=0}^{\infty}: f_{n} \in B\left(G_{n}\right),\|f\|_{B}:=\sup _{n \geq 0}\left\|f_{n}\right\|_{B\left(G_{n}\right)}<\infty\right\}
$$

Fix $x \in[0, \pi]$. Lemma 11 gives the following estimates:

$$
\left\|\varphi\left(x, g_{i}^{2}\right)\right\| \leq C, \quad\left\|\varphi\left(x, g_{i}^{2}\right)-\varphi\left(x, g_{j}^{2}\right)\right\| \leq C\left|g_{i}-g_{j}\right|, \quad g_{i}, g_{j} \in G_{n}
$$

where the constant $C$ does not depend on $n$. Therefore, $\varphi\left(x, \rho^{2}\right)$ forms an element of $B$ :

$$
\varphi\left(x, \rho^{2}\right)_{\mid B}:=\left\{\varphi\left(x, \rho^{2}\right)_{\mid G_{n}}\right\}_{n \geq 0} \in B, \quad \varphi\left(x, \rho^{2}\right)_{\mid G_{n}}=\left\{\varphi\left(x, \lambda_{n q i}\right)\right\}_{q=\overline{1, m}, i=0,1} .
$$

Denote $\psi(x):=\varphi\left(x, \rho^{2}\right)_{\mid B}, \tilde{\psi}(x):=\tilde{\varphi}\left(x, \rho^{2}\right)_{\mid B}$. Then (32) and (33) can be transformed into the following relations in the Banach space $B$ :

$$
\tilde{\psi}(x)=\psi(x)(I+\tilde{R}(x)),
$$




$$
\tilde{R}(x)-R(x)=R(x) \tilde{R}(x),
$$

where $I$ is the identity operator in $B$, and $R(x), \tilde{R}(x)$ are linear operators, acting from $B$ to $B .{ }^{4}$ The explicit form of $\tilde{R}(x)$ and $R(x)$ can be derived from (32) and (33). Further we investigate the operator $R(x)$, the same properties for $\tilde{R}(x)$ can be obtained symmetrically.

According to (32) and (33), the operator $R(x)$ acts on an arbitrary element $\psi=\left\{\psi_{k}\right\}_{k=0}^{\infty} \in B$ in the following way:

$$
\begin{aligned}
(\psi R(x))_{n} & =\sum_{k=0}^{\infty} \psi_{k} R_{k, n}(x), \quad R_{k, n}: B\left(G_{k}\right) \rightarrow B\left(G_{n}\right), \quad k, n \geq 0, \\
\left(\psi_{k} R_{k, n}(x)\right)\left(\rho_{n q i}\right) & =\sum_{l=1}^{m}\left(\psi_{k}\left(\rho_{k l 0}\right) \alpha_{k l 0}^{\prime} D\left(x, \lambda_{n q i}, \lambda_{k l 0}\right)-\psi_{k}\left(\rho_{k l 1}\right) \alpha_{k l 1}^{\prime} D\left(x, \lambda_{n q i}, \lambda_{k l 1}\right)\right) .
\end{aligned}
$$

Lemma 12. The series in (36) converge in $B\left(G_{n}\right)$-norm and the operator $R(x)$ is bounded and, moreover, compact on $B$.

Proof. Let $\psi=\left\{\psi_{k}\right\}_{k=0}^{\infty} \in B$. Fix $x \in[0, \pi]$ and $n, k \geq 0$. Denote $\psi_{k l j}:=\psi_{k}\left(\rho_{k l j}\right), \eta_{n q i, k}:=$ $\left(\psi_{k} R_{k, n}(x)\right)\left(\rho_{n q i}\right)$. Let us show that

$$
\left\|\eta_{n q i, k}\right\| \leq \frac{C \xi_{k}\left\|\psi_{k}\right\|_{B\left(G_{k}\right)}}{|n-k|+1}, \quad q=\overline{1, m}, i=0,1,
$$

where $\xi_{k}$ was defined in (28) and the constant $C$ does not depend on $n$ and $k$.

Using (37), we derive

$$
\begin{aligned}
\eta_{n q i, k}= & \sum_{l=1}^{m}\left[\left(\psi_{k l 0}-\psi_{k l 1}\right) \alpha_{k l 0}^{\prime} D\left(x, \lambda_{n q i}, \lambda_{k l 0}\right)\right. \\
& \left.+\psi_{k l 1} \alpha_{k l 0}^{\prime}\left(D\left(x, \lambda_{n q i}, \lambda_{k l 0}\right)-D\left(x, \lambda_{n q i}, \lambda_{k l 1}\right)\right)+\psi_{k l 1}\left(\alpha_{k l 0}^{\prime}-\alpha_{k l 1}^{\prime}\right) D\left(x, \lambda_{n q i}, \lambda_{k l 1}\right)\right] .
\end{aligned}
$$

Since

$$
\left\|\psi_{k l 1}\right\| \leq\left\|\psi_{k}\right\|_{B\left(G_{k}\right)}, \quad\left\|\psi_{k l 0}-\psi_{k l 1}\right\| \leq\left|\rho_{k l 0}-\rho_{k l 1}\right|\left\|\psi_{k}\right\|_{B\left(G_{k}\right)} \leq \xi_{k}\left\|\psi_{k}\right\|_{B\left(G_{k}\right)}, \quad l=\overline{1, m},
$$

$\left\|\alpha_{k l 0}^{\prime}\right\| \leq C$, and $D\left(x, \lambda_{n q i}, \lambda_{k l j}\right)$ satisfy estimates of Lemma 11 , one easily obtain the estimate (38) for the first two terms.

Recall that $J_{s}=\left\{q: \omega_{q}=\omega_{m_{s}}\right\}, s=\overline{1, p}$, are indices in groups with equal terms $\omega_{q}$ in asymptotics (3). Continue to work with the third term:

$$
\sum_{l=1}^{m} \psi_{k l 1}\left(\alpha_{k l 0}^{\prime}-\alpha_{k l 1}^{\prime}\right) D\left(x, \lambda_{n q i}, \lambda_{k l 1}\right)
$$

\footnotetext{
${ }^{4}$ The action of operators $R(x)$ and $\tilde{R}(x)$ is, in fact, a multiplication of an infinite row vector to an infinite matrix. It is more convenient to write operators to the right of operands, to keep the correct order in elementwise multiplication, which is the noncommutative multiplication of $m \times m$ matrices.
} 


$$
\begin{aligned}
= & \sum_{s=1}^{p}\left[\sum_{l \in J_{s}}\left(\psi_{k l 1}-\psi_{k m_{s} 1}\right)\left(\alpha_{k l 0}^{\prime}-\alpha_{k l 1}^{\prime}\right) D\left(x, \lambda_{n q i}, \lambda_{k l 1}\right)\right. \\
& +\sum_{l \in J_{s}} \psi_{k m_{s} 1}\left(\alpha_{k l 0}^{\prime}-\alpha_{k l 1}^{\prime}\right)\left(D\left(x, \lambda_{n q i}, \lambda_{k l 1}\right)-D\left(x, \lambda_{n q i}, \lambda_{k m_{s} 1}\right)\right) \\
& \left.+\psi_{k m_{s} 1}\left(\alpha_{k}^{(s)}-\tilde{\alpha}_{k}^{(s)}\right) D\left(x, \lambda_{n q i}, \lambda_{k m_{s} 1}\right)\right]
\end{aligned}
$$

Applying the estimate

$$
\left\|\psi_{k l 1}-\psi_{k m_{s} 1}\right\| \leq\left|\rho_{k l 1}-\rho_{k m_{s} 1}\right|\left\|\psi_{k}\right\|_{B\left(G_{k}\right)} \leq \xi_{k}\left\|\psi_{k}\right\|_{B\left(G_{k}\right)}, \quad l \in J_{s},
$$

estimates for $\alpha_{k l j}^{\prime}$ and Lemma 11, we arrive at (38) for the first two terms again and continue to investigate the third one.

$$
\begin{aligned}
& \sum_{s=1}^{p} \psi_{k m_{s} 1}\left(\alpha_{k}^{(s)}-\tilde{\alpha}_{k}^{(s)}\right) D\left(x, \lambda_{n q i}, \lambda_{k m_{s} 1}\right) \\
& =\sum_{s=1}^{p}\left(\psi_{k m_{s} 1}-\psi_{k 11}\right)\left(\alpha_{k}^{(s)}-\tilde{\alpha}_{k}^{(s)}\right) D\left(x, \lambda_{n q i}, \lambda_{k m_{s} 1}\right) \\
& \quad+\sum_{s=1}^{p} \psi_{k 11}\left(\alpha_{k}^{(s)}-\tilde{\alpha}_{k}^{(s)}\right)\left(D\left(x, \lambda_{n q i}, \lambda_{k m_{s} 1}\right)-D\left(x, \lambda_{n q i}, \lambda_{k 11}\right)\right)+\psi_{k 11}\left(\alpha_{k}-\tilde{\alpha}_{k}\right) D\left(x, \lambda_{n q i}, \lambda_{k 11}\right) .
\end{aligned}
$$

Now we use the estimates

$$
\begin{gathered}
\left\|\psi_{k m_{s} 1}-\psi_{k 11}\right\| \leq\left|\rho_{k m_{s} 1}-\rho_{k 11}\right|\left\|\psi_{k}\right\|_{B\left(G_{k}\right)} \leq \frac{\left\|\psi_{k}\right\|_{B\left(G_{k}\right)}}{k}, \\
\left\|\alpha_{k}^{(s)}-\tilde{\alpha}_{k}^{(s)}\right\| \leq k \xi_{k}, \quad\left\|\alpha_{k}-\tilde{\alpha}_{k}\right\| \leq \xi_{k},
\end{gathered}
$$

(following from (7), (9) and similar asymptotics for $\tilde{\alpha}_{n q}$ ) and Lemma 11. Finally we arrive at (38).

Analogously one can obtain the estimate

$$
\left\|\eta_{n q i, k}-\eta_{n l j, k}\right\| \leq \frac{C \xi_{k}\left\|\psi_{k}\right\|_{B\left(G_{k}\right)}\left|\rho_{n q i}-\rho_{n l j}\right|}{|n-k|+1}, \quad q, l=\overline{1, m}, i, j=0,1 .
$$

Together with (38), this gives

$$
\left\|R_{k, n}(x)\right\|_{B\left(G_{k}\right) \rightarrow B\left(G_{n}\right)} \leq \frac{C \xi_{k}}{|n-k|+1}, \quad k, n \geq 0,
$$

where the constant $C$ does not depend on $n$ and $k$. Substitute (39) into (36) and use (29):

$$
\|\psi R(x)\|_{B}=\sup _{n \geq 0}\left\|(\psi R(x))_{n}\right\| \leq\|\psi\|_{B}\left(\sum_{k=0}^{\infty} \frac{C \xi_{k}}{|n-k|+1}\right) \leq C\|\psi\|_{B} .
$$

Hence $\|R(x)\|_{B \rightarrow B}<\infty$.

The operator $R(x)$ can be approximated by a sequence of finite-dimensional operators in $B$. Indeed, let $R_{k, n}^{s}(x)=R_{k, n}(x)$ for all $n \geq 0,0 \leq k \leq s$, and all the other components of $R^{s}(x)$ equal zero. It is easy to show using (39), that $\lim _{s \rightarrow \infty}\left\|R^{s}(x)-R(x)\right\|_{B \rightarrow B}=0$. Therefore the operator $R(x)$ is compact. 
Theorem 4. For each fixed $x \in[0, \pi]$, the operator $I+\tilde{R}(x)$ has a bounded inverse operator, and equation (34) is uniquely solvable in the Banach space $B$.

Proof. It follows from (35), that for each fixed $x \in[0, \pi],(I-R(x))(I+\tilde{R}(x))=I$. Symmetrically, one gets $(I+\tilde{R}(x))(I-R(x))=I$. Hence the operator $(I+\tilde{R}(x))^{-1}$ exists, and it is a linear bounded operator by Lemma 12 .

Equation (34) is called the main equation of Inverse Problem 1. Theorem 4 together with Lemmas 1, 2, 3 and 6 gives the necessity part in Theorem 1.

Now turn to the problem $L^{*}$, defined in (11). Take the model problem $\tilde{L}^{*}=L^{*}(\tilde{Q}(x), \tilde{h}, \tilde{H})$ with the same potential $\tilde{Q}$ as the problem $\tilde{L}$ has. By virtue of (16), the problems $L$ and $L^{*}$ (similarly, $\tilde{L}$ and $\tilde{L}^{*}$ ) have the same spectral data. Symmetrically to (32), we obtain the relations

$$
\tilde{\varphi}_{n q i}^{*}(x)=\varphi_{n q i}^{*}(x)+\sum_{k=0}^{\infty} \sum_{l=1}^{m}\left(\tilde{D}\left(x, \lambda_{k l 0}, \lambda_{n q i}\right) \alpha_{k l 0}^{\prime} \varphi_{k l 0}^{*}(x)-\tilde{D}\left(x, \lambda_{k l 1}, \lambda_{n q i}\right) \alpha_{k l 1}^{\prime} \varphi_{k l 1}^{*}(x)\right),
$$

for each fixed $x \in[0, \pi], n \geq 0, q=\overline{1, m}, i=0,1$. Similarly to $B$, introduce the Banach space $B^{*}$ of column vectors. Then $\psi^{*}=\left\{\psi_{k}^{*}\right\}_{k=0}^{\infty}, \psi_{k}^{*}=\left[\varphi_{k l j}^{*}(x)\right]_{l=\overline{1, m}, j=0,1}$ satisfy the linear equation

$$
\left(I+\tilde{R}^{*}(x)\right) \psi^{*}(x)=\tilde{\psi}^{*}(x)
$$

in $B^{*}$ for each fixed $x \in[0, \pi]$. Here $\tilde{\psi}^{*}(x)$ and $\tilde{R}^{*}(x)$ are constructed symmetrically to $\tilde{\psi}(x)$ and $\tilde{R}(x)$ by the model problem $\tilde{L}^{*}$ and the spectral data $\Lambda, \tilde{\Lambda}$.

Lemma 13. For each fixed $x \in[0, \pi]$, equation (41) is uniquely solvable in the Banach space $B^{*}$ if and only if equation (34) is uniquely solvable.

Proof. Fix $x \in[0, \pi]$. In view of Lemma 12 , the operators $\tilde{R}(x)$ and $\tilde{R}^{*}(x)$ are compact in the corresponding Banach spaces. Therefore it is sufficient to consider homogeneous equations $\gamma(x)(I+\tilde{R}(x))=0$ and $\left(I+\tilde{R}^{*}(x)\right) \gamma^{*}(x)=0$. Let us prove only the "if" part, since the "only if" part can be proved symmetrically.

Suppose the equation $\gamma(x)(I+\tilde{R}(x))=0$ is uniquely solvable. Then there exists a bounded inverse operator $\tilde{P}(x)=(I+\tilde{R}(x))^{-1}$ of the following form

$$
\begin{gathered}
(\psi \tilde{P}(x))_{n q i}=\sum_{k=0}^{\infty} \sum_{l=1}^{m}\left(\psi_{k l 0} \tilde{P}_{k l 0, n q i}(x)-\psi_{k l 1} \tilde{P}_{k l 1, n q i}(x)\right), \\
\tilde{P}(x)=\left[\tilde{P}_{k, n}(x)\right]_{k, n \geq 0}=\left[\tilde{P}_{k l j, n q i}(x)\right], \quad \psi=\left[\psi_{k l j}\right] \in B, \quad n, k \geq 0 q, l=\overline{1, m}, i, j=0,1 .
\end{gathered}
$$

It follows from $(\psi \tilde{P}(x)) \in B$, that

$$
\left\|\tilde{P}_{r s t, n q i}(x)-\tilde{P}_{r s t, n l j}(x)\right\| \leq C\left|\rho_{n q i}-\rho_{n l j}\right|, \quad r, n \geq 0, s, q, l=\overline{1, m}, t, i, j=0,1 .
$$


For simplicity, assume that all the values $\left\{\lambda_{n q i}\right\}$ are distinct (the general case requires minor modifications). The relation $\tilde{P}(x)(I+\tilde{R}(x))=I$ yields

$$
\begin{gathered}
\tilde{P}_{r s t, n q i}(x)+\sum_{k=0}^{\infty} \sum_{l=1}^{n}\left(\tilde{P}_{r s t, k l 0}(x) \alpha_{k l 0}^{\prime} \tilde{D}\left(x, \lambda_{n q i}, \lambda_{k l 0}\right)-\tilde{P}_{r s t, k l 1}(x) \alpha_{k l 1}^{\prime} \tilde{D}\left(x, \lambda_{n q i}, \lambda_{k l 1}\right)\right)=\delta_{r s t, n q i}, \\
n, r \geq 0, \quad s, q=\overline{1, m}, \quad t, i=0,1,
\end{gathered}
$$

where $\delta_{r s t, n q i}=I_{m}$, if $(r, s, t)=(n, q, i)$, and $\delta_{r s t, n q i}=0_{m}$ otherwise.

Let $\gamma^{*}(x)=\left[\gamma_{n q i}^{*}(x)\right]$ be a solution of the equation $\left(I+\tilde{R}^{*}(x)\right) \gamma^{*}(x)=0$ :

$$
\gamma_{n q i}^{*}(x)+\sum_{k=0}^{\infty} \sum_{l=1}^{m}\left(\tilde{D}\left(x, \lambda_{k l 0}, \lambda_{n q i}\right) \alpha_{k l 0}^{\prime} \gamma_{k l 0}^{*}(x)-\tilde{D}\left(x, \lambda_{k l 1}, \lambda_{n q i}\right) \alpha_{k l 1}^{\prime} \gamma_{k l 1}^{*}(x)\right)=0_{m},
$$

$n \geq 0, q=\overline{1, m}, i=0,1$. Then

$\sum_{n=0}^{\infty} \sum_{q=1}^{m} \sum_{i=0}^{1}(-1)^{i} \tilde{P}_{r s t, n q i}(x) \alpha_{n q i}^{\prime} \gamma_{n q i}^{*}(x)$

$+\sum_{n, k=0}^{\infty} \sum_{q, l=1}^{m} \sum_{i, j=0}^{1}(-1)^{i+j} \tilde{P}_{r s t, n q i}(x) \alpha_{n q i}^{\prime} \tilde{D}\left(x, \lambda_{k l j}, \lambda_{n q i}\right) \alpha_{k l j}^{\prime} \gamma_{k l j}^{*}(x)=0_{m}, r \geq 0, s=\overline{1, m}, t=0,1$.

Convergence of the series can be proved with help of (42). Using (43), we obtain

$\sum_{n=0}^{\infty} \sum_{q=1}^{m} \sum_{i=0}^{1}(-1)^{i} \tilde{P}_{r s t, n q i}(x) \alpha_{n q i}^{\prime} \gamma_{n q i}^{*}(x)+\sum_{k=0}^{\infty} \sum_{l=1}^{m} \sum_{j=0}^{1}(-1)^{j}\left(\delta_{r s t, k l j}-\tilde{P}_{r s t, k l j}(x)\right) \alpha_{k l j}^{\prime} \gamma_{k l j}^{*}(x)=0_{m}$.

Consequently, $\alpha_{k l j}^{\prime} \gamma_{k l j}^{*}(x)=0_{m}$ for all $k \geq 0, l=\overline{1, m}, j=0,1$. In view of (44), we conclude that $\gamma^{*}(x)=0$, so the homogeneous equation $\left(I+\tilde{R}^{*}(x)\right) \gamma^{*}(x)=0$ is uniquely solvable.

Note that in the proof of Lemma 13 we do not use the fact, that $\Lambda$ is the spectral data of $L$, but use only properties (A) and (R). Therefore this lemma can be used in the sufficiency part.

The main equation gives us a constructive solution of Inverse problem 1. Solving (34), we find the vector $\psi(x)$, i.e. the matrix-functions $\varphi_{n q i}(x)$.

Denote

$$
\varepsilon_{0}(x)=\sum_{k=0}^{\infty} \sum_{l=1}^{m}\left(\varphi_{k l 0}(x) \alpha_{k l 0}^{\prime} \tilde{\varphi}_{k l 0}^{*}(x)-\varphi_{k l 1}(x) \alpha_{k l 1}^{\prime} \tilde{\varphi}_{k l 1}^{*}(x)\right), \quad \varepsilon(x)=-2 \varepsilon_{0}^{\prime}(x) .
$$

Lemma 14. The series in (45) converges absolutely and uniformly on $[0, \pi]$, the function $\varepsilon_{0}(x)$ is absolutely continuous, and $\varepsilon(x) \in L_{2}\left((0, \pi), \mathbb{C}^{m \times m}\right)$.

Proof. Here we use ideas similar to the proof of Lemma 12. Group the terms of (45) in the following way:

$$
\sum_{l=1}^{m}\left(\varphi_{k l 0}(x) \alpha_{k l 0}^{\prime} \tilde{\varphi}_{k l 0}^{*}(x)-\varphi_{k l 1}(x) \alpha_{k l 1}^{\prime} \tilde{\varphi}_{k l 1}^{*}(x)\right)
$$




$$
\begin{aligned}
= & \sum_{l=1}^{m}\left(\varphi_{k l 0}(x)-\varphi_{k l 1}(x)\right) \alpha_{k l 0}^{\prime} \tilde{\varphi}_{k l 0}^{*}(x)+\sum_{l=1}^{m} \varphi_{k l 1}(x) \alpha_{k l 0}^{\prime}\left(\tilde{\varphi}_{k l 0}^{*}(x)-\tilde{\varphi}_{k l 1}^{*}(x)\right) \\
& +\sum_{s=1}^{p} \sum_{l \in J_{s}}\left(\varphi_{k l 1}(x)-\varphi_{k m_{s} 1}(x)\right)\left(\alpha_{k l 0}^{\prime}-\alpha_{k l 1}^{\prime}\right) \tilde{\varphi}_{k l 1}^{*}(x) \\
& +\sum_{s=1}^{p} \sum_{l \in J_{s}} \varphi_{k m_{s} 1}(x)\left(\alpha_{k l 0}^{\prime}-\alpha_{k l 1}^{\prime}\right)\left(\tilde{\varphi}_{k l 1}^{*}(x)-\tilde{\varphi}_{k m_{s} 1}^{*}(x)\right) \\
& +\sum_{s=1}^{p}\left(\varphi_{k m_{s} 1}(x)-\varphi_{k 11}(x)\right)\left(\alpha_{k}^{(s)}-\tilde{\alpha}_{k}^{(s)}\right) \tilde{\varphi}_{k m_{s} 1}^{*}(x) \\
& +\sum_{s=1}^{p} \varphi_{k 11}(x)\left(\alpha_{k}^{(s)}-\tilde{\alpha}_{k}^{(s)}\right)\left(\tilde{\varphi}_{k m_{s} 1}^{*}(x)-\tilde{\varphi}_{k 11}^{*}(x)\right)+\varphi_{k 11}(x)\left(\alpha_{k}-\tilde{\alpha}_{k}\right) \tilde{\varphi}_{k 11}^{*}(x) .
\end{aligned}
$$

It follows from (28), (29) and Lemma 11, that the series in (45) converges absolutely and uniformly on $[0, \pi]$ :

$$
\left\|\varepsilon_{0}(x)\right\| \leq C \sum_{k=0}^{\infty} \xi_{k}<\infty .
$$

Let us analyze the derivative of the first term in (46):

$$
\begin{aligned}
S^{\prime}(x) & :=\frac{d}{d x}\left(\left(\varphi_{k l 0}(x)-\varphi_{k l 1}(x)\right) \alpha_{k l 0}^{\prime} \tilde{\varphi}_{k l 0}^{*}(x)\right) \\
& =\left(\varphi_{k l 0}^{\prime}(x)-\varphi_{k l 1}^{\prime}(x)\right) \alpha_{k l 0}^{\prime} \tilde{\varphi}_{k l 0}^{*}(x)+\left(\varphi_{k l 0}(x)-\varphi_{k l 1}(x)\right) \alpha_{k l 0}^{\prime}{\tilde{\varphi^{*}}}_{k l 0}^{\prime}(x)
\end{aligned}
$$

The other terms can be treated similarly. Using asymptotics (3), (21) and (22) together with Schwarz's lemma, one gets

$$
\begin{array}{ll}
\varphi_{k l 0}^{\prime}(x)-\varphi_{k l 1}^{\prime}(x)=-\cos k x \gamma_{k l} x I_{m}+\frac{K_{k}(x)}{k+1}, & \tilde{\varphi}_{k l 0}^{*}(x)=\cos k x+O\left(k^{-1}\right), \\
\varphi_{k l 0}(x)-\varphi_{k l 1}(x)=-\sin k x \frac{\gamma_{k l}}{k+1} x I_{m}+\frac{K_{k}(x)}{(k+1)^{2}}, & \tilde{\varphi}_{k l 0}^{* \prime}(x)=-k \sin k x+O(1),
\end{array}
$$

where $\gamma_{k l}=(k+1)\left(\rho_{k l 0}-\rho_{k l 1}\right),\left\{\gamma_{k l}\right\} \in l_{2}, K_{k}(x)$ denotes various sequences of matrix functions, continuous on $[0, \pi]$, such that $\left\{\max _{x}\left\|K_{k}(x)\right\|\right\} \in l_{2}$. Then

$$
S^{\prime}(x)=-\cos 2 k x \gamma_{k l} x \alpha_{k l 0}^{\prime}+\frac{K_{k}(x)}{k+1}
$$

By the Riesz-Fischer theorem,

$$
x \sum_{k=0}^{\infty} \cos 2 k x \gamma_{k l} \alpha_{k l 0}^{\prime} \in L_{2}\left((0, \pi), \mathbb{C}^{m \times m}\right) .
$$

The series $\sum_{k=0}^{\infty} \frac{K_{k}(x)}{k+1}$ converges absolutely and uniformly with respect to $x \in[0, \pi]$. Hence $\varepsilon(x) \in L_{2}\left((0, \pi), \mathbb{C}^{m \times m}\right)$.

The next lemma gives formulas for recovering the potential $Q(x)$ and the coefficients of the boundary conditions $h$ and $H$. 
Lemma 15. The following relations hold

$$
Q(x)=\tilde{Q}(x)+\varepsilon(x), \quad h=\tilde{h}-\varepsilon_{0}(0), \quad H=\tilde{H}+\varepsilon_{0}(\pi),
$$

Proof. The proof is similar to [11, Lemma 8]. Differentiating (30) twice with respect to $x$ and using (27) and (45) we get

$$
\begin{aligned}
\tilde{\varphi}^{\prime}(x, \lambda)-\varepsilon_{0}(x) \tilde{\varphi}(x, \lambda)= & \varphi^{\prime}(x, \lambda)+\sum_{k=0}^{\infty} \sum_{l=1}^{m} \sum_{j=0}^{1}(-1)^{j} \varphi_{k l j}^{\prime}(x) \alpha_{k l j}^{\prime} \tilde{D}\left(x, \lambda, \lambda_{k l j}\right) \\
\tilde{\varphi}^{\prime \prime}(x, \lambda)= & \varphi^{\prime \prime}(x, \lambda)+\sum_{k=0}^{\infty} \sum_{l=1}^{m} \sum_{j=0}^{1}(-1)^{j}\left[\varphi_{k l j}^{\prime \prime}(x) \alpha_{k l j}^{\prime} \tilde{D}\left(x, \lambda, \lambda_{k l j}\right)\right. \\
& \left.+2 \varphi_{k l j}^{\prime}(x) \alpha_{k l j}^{\prime} \tilde{\varphi}_{k l j}^{*}(x) \tilde{\varphi}(x, \lambda)+\varphi_{k l j}(x) \alpha_{k l j}^{\prime}\left(\tilde{\varphi}_{k l j}^{*}(x) \tilde{\varphi}(x, \lambda)\right)^{\prime}\right] .
\end{aligned}
$$

We replace here the second derivatives, using equation (1), and then replace $\varphi(x, \lambda)$, using (30). This yields

$$
\begin{aligned}
\tilde{Q}(x) \varphi(x, \lambda)= & Q(x) \tilde{\varphi}(x, \lambda)+\sum_{k=0}^{\infty} \sum_{l=1}^{m} \sum_{j=0}^{1}(-1)^{j}\left[\varphi_{k l j}(x) \alpha_{k l j}^{\prime}\left\langle\tilde{\varphi}_{k l j}^{*}(x), \tilde{\varphi}(x, \lambda)\right\rangle\right. \\
& \left.+2 \varphi_{k l j}^{\prime}(x) \alpha_{k l j}^{\prime} \tilde{\varphi}_{k l j}^{*}(x) \tilde{\varphi}(x, \lambda)+\varphi_{k l j}(x) \alpha_{k l j}^{\prime}\left(\tilde{\varphi}_{k l j}^{*}(x) \tilde{\varphi}(x, \lambda)\right)^{\prime}\right] .
\end{aligned}
$$

Cancelling terms with $\tilde{\varphi}^{\prime}(x, \lambda)$ we arrive at $Q(x)=\tilde{Q}(x)+\varepsilon(x)$.

Further,

$$
\tilde{\varphi}^{\prime}(0, \lambda)-\left(h+\varepsilon_{0}(0)\right) \tilde{\varphi}(0)=U(\varphi)+\sum_{k=0}^{\infty} \sum_{l=1}^{m} \sum_{j=0}^{1}(-1)^{j} U\left(\varphi_{k l j}\right) \alpha_{k l j}^{\prime} D\left(0, \lambda, \lambda_{k l j}\right)=0_{m} .
$$

Since $\tilde{\varphi}(0, \lambda)=I_{m}, \tilde{\varphi}^{\prime}(0, \lambda)=\tilde{h}$, we obtain $h=\tilde{h}-\varepsilon_{0}(0)$.

Similarly, using (31) one can get

$$
\tilde{\Phi}^{\prime}(\pi, \lambda)+\left(H-\varepsilon_{0}(\pi)\right) \Phi(\pi, \lambda)=V(\Phi)+\sum_{k=0}^{\infty} \sum_{l=1}^{m} \sum_{j=0}^{1}(-1)^{j} V\left(\varphi_{k l j}\right) \alpha_{k l j}^{\prime} \frac{\left\langle\tilde{\varphi}_{k l j}^{*}(x), \tilde{\Phi}(x, \lambda)\right\rangle_{\mid x=\pi}}{\lambda-\lambda_{k l j}} .
$$

For $j=0$ we have $V\left(\varphi_{k l 0}\right) \alpha_{k l 0}^{\prime}=0_{m}$. For $j=1$

$$
\left\langle\tilde{\varphi}_{k l 1}^{*}(x), \tilde{\Phi}(x, \lambda)\right\rangle_{\mid x=\pi}=\tilde{V}^{*}\left(\tilde{\varphi}_{k l 1}^{*}\right) \tilde{\Phi}(\pi, \lambda)-\tilde{\varphi}_{k l 1}^{*}(\pi) \tilde{V}(\tilde{\Phi}) .
$$

Recall that $V(\Phi)=0_{m}, \tilde{V}(\tilde{\Phi})=0_{m}$ and $\alpha_{k l 1}^{\prime} \tilde{V}^{*}\left(\tilde{\varphi}_{k l 1}^{*}\right)=0_{m}$. Consequently, we arrive at $\tilde{\Phi}^{\prime}(\pi, \lambda)+$ $\left(H-\varepsilon_{0}(\pi)\right) \Phi(\pi, \lambda)=0_{m}$. Together with $\tilde{V}(\tilde{\Phi})=0_{m}$ this yields $H=\tilde{H}+\varepsilon(\pi)$.

Thus, we obtain the following algorithm for the solution of Inverse Problem 1.

Algorithm 1. Given the data $\Lambda$. 
1. Choose $\tilde{L} \in A(\omega)$, and calculate $\tilde{\psi}(x)$ and $\tilde{R}(x)$.

2. Find $\psi(x)$ by solving equation (34), and calculate $\varphi_{n q i}(x)$.

3. Construct $Q(x), h$ and $H$ by (47).

\section{Conditions (M), (E), (C) and (PW)}

In this section, we establish the connection between the solvability of the main equation $(\mathrm{M})$, the condition (E) and the completeness of some system of functions (C). The condition (C) and its equivalent reformulation (PW) will be given further in this section.

Let $\omega \in \mathscr{D}$ and data $\Lambda \in$ Sp satisfy conditions (A) and (R) of Theorem 1. Let $\tilde{L}$ be an arbitrary problem from the class $A_{1,2}(\omega)$.

Lemma 16. (E) follows from (M).

Proof. Let $\gamma(\lambda)$ be a row vector, entire in $\lambda$ and satisfying the relations $\gamma(\lambda)=O(\exp (|\tau| \pi))$, $\gamma\left(\lambda_{n q 0}\right) \alpha_{n q 0}=0$ for all $n \geq 0, q=\overline{1, m}$.

Schwarz's lemma together with asymptotics (3) yields

$$
\left\|\gamma\left(\lambda_{n q i}\right)-\gamma\left(\lambda_{n l j}\right)\right\| \leq C\left|\rho_{n q i}-\rho_{n l j}\right|, \quad n \geq 0, q, l=\overline{1, m}, i, j=0,1 .
$$

Consider the function

$$
\tilde{\gamma}(\lambda):=\gamma(\lambda)+\sum_{k=0}^{\infty} \sum_{l=1}^{\infty}\left[\gamma\left(\lambda_{k l 0}\right) \alpha_{k l 0}^{\prime} \tilde{D}\left(\pi, \lambda, \lambda_{k l 0}\right)-\gamma\left(\lambda_{k l 1}\right) \alpha_{k l 1}^{\prime} \tilde{D}\left(\pi, \lambda, \lambda_{k l 1}\right)\right]
$$

In order to prove the convergence of the series in (49), we apply the following formal transformation

$$
\begin{aligned}
\tilde{\gamma}(\lambda)= & \gamma(\lambda)+\sum_{k=0}^{\infty}\left[\sum_{l=1}^{m}\left(\gamma\left(\lambda_{k l 0}\right)-\gamma\left(\lambda_{k l 1}\right)\right) \alpha_{k l 0}^{\prime} \tilde{D}\left(\pi, \lambda, \lambda_{k l 0}\right)\right. \\
& +\sum_{l=1}^{m} \gamma\left(\lambda_{k l 1}\right) \alpha_{k l 0}^{\prime}\left(\tilde{D}\left(\pi, \lambda, \lambda_{k l 0}\right)-\tilde{D}\left(\pi, \lambda, \lambda_{k l 1}\right)\right) \\
& +\sum_{s=1}^{p} \sum_{l \in J_{s}}\left(\gamma\left(\lambda_{k l 1}\right)-\gamma\left(\lambda_{k m_{s} 1}\right)\right)\left(\alpha_{k l 0}^{\prime}-\alpha_{k l 1}^{\prime}\right) \tilde{D}\left(\pi, \lambda, \lambda_{k l 1}\right) \\
& +\sum_{s=1}^{p} \sum_{l \in J_{s}} \gamma\left(\lambda_{k m_{s} 1}\right)\left(\alpha_{k l 0}^{\prime}-\alpha_{k l 1}^{\prime}\right)\left(\tilde{D}\left(\pi, \lambda, \lambda_{k l 1}\right)-\tilde{D}\left(\pi, \lambda, \lambda_{k m_{s} 1}\right)\right) \\
& +\sum_{s=1}^{p}\left(\gamma\left(\lambda_{k m_{s} 1}\right)-\gamma\left(\lambda_{k 11}\right)\right)\left(\alpha_{k}^{(s)}-\tilde{\alpha}_{k}^{(s)}\right) \tilde{D}\left(\pi, \lambda, \lambda_{k m_{s} 1}\right) \\
& +\sum_{s=1}^{p} \gamma\left(\lambda_{k 11}\right)\left(\alpha_{k}^{(s)}-\tilde{\alpha}_{k}^{(s)}\right)\left(\tilde{D}\left(\pi, \lambda, \lambda_{k m_{s} 1}\right)-\tilde{D}\left(\pi, \lambda, \lambda_{k 11}\right)\right)
\end{aligned}
$$




$$
\left.+\gamma\left(\lambda_{k 11}\right)\left(\alpha_{k}-\tilde{\alpha}_{k}\right) \tilde{D}\left(\pi, \lambda, \lambda_{k 11}\right)\right]
$$

By virtue of (28), (48) and the estimates

$$
\begin{gathered}
\left\|\tilde{D}\left(\pi, \lambda, \lambda_{k l j}\right)\right\| \leq C \exp (|\tau| \pi), \quad \| \tilde{D}\left(\pi, \lambda, \lambda_{k l j}\right)-\tilde{D}\left(x, \lambda, \lambda_{k q i} \| \leq C\left|\rho_{k l j}-\rho_{k q i}\right| \exp (|\tau| \pi),\right. \\
\operatorname{Re} \rho \geq 0, k \geq 0, l, q=\overline{1, m}, i, j=0,1,
\end{gathered}
$$

we get

$$
\|\tilde{\gamma}(\lambda)\| \leq\|\gamma(\lambda)\|+C \exp (|\tau| \pi) \sum_{k=0}^{\infty} \xi_{k}
$$

Taking (29) into account, we conclude that the series in (49) converges to an entire function, satisfying the estimate $\tilde{\gamma}(\lambda)=O(\exp (|\tau| \pi))$.

Substitute $\lambda=\lambda_{n q 1}$ into (49) and multiply the result by $\alpha_{n q 1}$. Definition (27) and Lemma 7 yield

$$
\alpha_{k l 1}^{\prime} \tilde{D}\left(\pi, \lambda_{n q 1}, \lambda_{k l 1}\right) \alpha_{n q 1}=\left\{\begin{array}{l}
\alpha_{n q 1}, \quad \text { if } \alpha_{n q 1}=\alpha_{k l 1} \text { and } \alpha_{k l 1}^{\prime} \neq 0_{m} \\
0_{m}, \quad \text { otherwise. }
\end{array}\right.
$$

Therefore, we obtain $\tilde{\gamma}\left(\lambda_{n q 1}\right) \alpha_{n q 1}=0$ for all $n \geq 0, q=\overline{1, m}$. Thus, we have got the entire function $\tilde{\gamma}(\lambda)$, satisfying the presupposition of (E) for the spectral data $\tilde{\Lambda}$ of the model problem $\tilde{L}$. But (E) holds for $\tilde{\Lambda}$ by Lemma 8 . Hence $\tilde{\gamma}(\lambda) \equiv 0$, and by (49)

$$
\gamma\left(\lambda_{n q i}\right)+\sum_{k=0}^{\infty} \sum_{l=1}^{m}\left(\gamma\left(\lambda_{k l 0}\right) \alpha_{k l 0}^{\prime} \tilde{D}\left(\pi, \lambda_{n q i}, \lambda_{k l 0}\right)-\gamma\left(\lambda_{k l 1}\right) \alpha_{k l 1}^{\prime} \tilde{D}\left(\pi, \lambda_{n q i}, \lambda_{k l 1}\right)\right)=0 .
$$

We see that the vector $\psi=\left[\gamma\left(\lambda_{n q i}\right)\right] \in B$ satisfy the homogeneous main equation $\psi(I+\tilde{R}(\pi))=$ 0 . It follows from (M), that $\gamma\left(\lambda_{n q i}\right)=0$ for all $n \geq 0, q=\overline{1, m}, i=0,1$. Using (49) once again, we arrive at $\gamma(\lambda) \equiv 0$. Thus, we have proved (E).

Introduce the subspaces $\mathscr{E}_{n q}=\operatorname{Ran} \alpha_{n q}^{\prime}=\left\{\mathscr{E}=\alpha_{n q}^{\prime} h, h \in \mathbb{C}^{m}\right\}$. Note that we intendently use $\alpha_{n q}^{\prime}$ instead of $\alpha_{n q}$ in this definition, in order not to include the same subspaces multiple times. Let $\left\{\mathscr{E}_{n q}^{(i)}\right\}_{i=1}^{m_{n q}}$ be an ortonormal basis of $\mathscr{E}_{n q}$. The number $m_{n q}$ coincide with the multiplicity of the corresponding eigenvalue $\lambda_{n q}$, if $\alpha_{n q}^{\prime} \neq 0_{m}$, and $\mathscr{E}_{n q}=\varnothing$ otherwise.

Lemma 17. Let $\varphi(x, \lambda)$ be an arbitrary matrix-function, continuous with respect to $x \in[0, \pi]$ and entire in $\lambda$, satisfying the asymptotic relation (21). Suppose that (E) holds. Then the system

$$
\varphi\left(x, \lambda_{n q}\right) \mathscr{E}_{n q}^{(i)}, \quad n \geq 0, q=\overline{1, m}, i=\overline{1, m_{n q}},
$$

is complete in $L_{2}\left((0, \pi), \mathbb{C}^{m}\right)$. 
Proof. Consider a vector-function $f(x) \in L_{2}\left((0, \pi), \mathbb{C}^{m}\right)$, such that

$$
\int_{0}^{\pi} f^{\dagger}(x) \varphi\left(x, \lambda_{n q}\right) \mathscr{E}_{n q}^{(i)} d x=0
$$

for all $n \geq 0, q=\overline{1, m}, i=\overline{1, m_{n q}}$. It is easy to check that the function

$$
\gamma(\lambda)=\int_{0}^{\pi} f^{\dagger}(x) \varphi(x, \lambda) d x
$$

satisfy all the properties in (E) and, consequently, equals zero. Therefore $f(x)=0$ and the system (52) is complete.

Lemma 18. Let $\varphi(x, \lambda)$ satisfy conditions of Lemma 17, and the system (52) is complete. Then the system $(52)$ is a basis in $L_{2}\left((0, \pi), \mathbb{C}^{m}\right)$.

Proof. The basis property of the system (52) follows from its completeness and $l_{2}$-closeness to the ortonormal basis

$$
\cos n x \mathscr{E}_{n q}^{(i)}, \quad n \geq 0, q=\overline{1, m}, i=\overline{1, m_{n q}},
$$

According to asymptotics (3) and (21),

$$
\cos n x I_{m}-\varphi\left(x, \lambda_{n q}\right)=O\left(n^{-1}\right), \quad\left\{\left\|\left(\cos n x I_{m}-\varphi\left(x, \lambda_{n q}\right)\right) \mathscr{E}_{n q}^{(i)}\right\|\right\} \in l_{2} .
$$

In order to prove the basis property for the system (53), it is sufficient to show, that the system $\left\{\mathscr{E}_{n q}^{(i)}\right\}$ is a basis in $\mathbb{C}^{m}$ for a fixed $n$, for all sufficiently large values of $n \geq N$. If the elements of (53) for $n<N$ are linearly dependent, one can change them to $\cos n x e_{q}, q=\overline{1, m}$, where $\left\{e_{q}\right\}_{q=1}^{m}$ is the standard coordinate basis. Since (R) holds, i.e. ranks of the weight matrices equal multiplicities of the corresponding eigenvalues, the total number of the vectors $\left\{\mathscr{E}_{n q}^{(i)}\right\}$ for a fixed $n$ is $m$. Suppose there exists a vector $h$ ortogonal to all $\mathscr{E}_{n q}^{(i)}$. Then $h^{\dagger} \alpha_{n q}=0$ for all $q=\overline{1, m}$, and $h^{\dagger} \alpha_{n}=0$. But in view of (9), $\operatorname{det} \alpha_{n} \neq 0$ for sufficiently large values of $n$. Thus, the considered system of vectors is a basis.

Similar facts can be obtained for the problem $L^{*}$. Let $\mathscr{E}_{n q}^{*}=\left\{\mathscr{E}^{*}=h \alpha_{n q}^{\prime}, h \in \mathbb{C}^{m, T}\right\}$. Denote by $\left\{\mathscr{E}_{n q}^{*,(i)}\right\}_{i=1}^{m_{n q}}$ an ortonormal basis of $\mathscr{E}_{n q}^{*}$, consisting of row vectors. The following lemma summarizes results, similar to Lemmas 16-18. In fact, the solvability of the main equation (41) for $L^{*}$, instead of (34), can be used to prove the lemma.

Lemma 19. Assume that $\varphi(x, \lambda)$ is an arbitrary matrix-function, continuous with respect to $x \in[0, \pi]$ and entire in $\lambda$, satisfying the asymptotic relation (21), and (M) holds. Then the system

$$
\mathscr{E}_{n q}^{(i), *} \varphi\left(x, \lambda_{n q}\right), \quad n \geq 0, q=\overline{1, m}, i=\overline{1, m_{n q}},
$$

is a basis in $L_{2}\left((0, \pi), \mathbb{C}^{m, T}\right)$. 
By virtue of Lemma 17, the following condition:

(C) The system of functions

$$
\cos \rho_{n q} x \mathscr{E}_{n q}^{(i)}, \quad n \geq 0, q=\overline{1, m}, i=\overline{1, m_{n q}},
$$

is complete in $L_{2}\left((0, \pi), \mathbb{C}^{m}\right)$.

Follows from (E). The converse is not true.

Indeed, there is the one-to-one correspondence between vector-functions $f(x) \in L_{2}\left((0, \pi), \mathbb{C}^{m}\right)$ and row vectors $\gamma(\lambda)=\int_{0}^{\pi} f^{\dagger}(x) \cos \rho x d x$ of an even Paley-Wiener class PW, defined by the following conditions:

1. $\gamma(\lambda)$ is entire,

2. $\gamma(\lambda)=O(\exp (|\tau| \pi))$,

3. $\int_{0}^{\infty}\left|\gamma\left(\rho^{2}\right)\right|^{2} d \rho<\infty$.

Therefore, condition (C) is equivalent to the following condition:

(PW) For any row vector $\gamma(\lambda) \in P W$, if $\gamma\left(\lambda_{n q}\right) \alpha_{n q}=0$ for all $n \geq 0, q=\overline{1, m}$, then $\gamma(\lambda) \equiv 0$.

\section{Proof of Theorem 1}

The necessity in Theorem 1 is contained in Lemmas 1, 2, 3, 6 and Theorem 4 .

Turn to the proof of the sufficiency. Let data $\left\{\lambda_{n q}, \alpha_{n q}\right\}_{n \geq 0, q=\overline{1, m}} \in$ Sp be given. Choose $\tilde{L} \in$ $A_{1,2}(\omega)$ and construct $\tilde{\psi}(x), \tilde{R}(x)$. Assume that the conditions of Theorem 1 hold. Let $\psi(x)=$ $\left\{\psi_{n}(x)\right\}_{n \geq 0} \in B$ be the unique solution of the main equation (34). The proofs of Lemmas 20-22 are analogous to ones described in [4, Sec. 1.6.2].

Lemma 20. For $n \geq 0$, the functions $\psi_{n}(x)$ are continuously differentiable with respect to $x$ on $[0, \pi]$, and the following relations hold

$$
\begin{gathered}
\left\|\psi_{n}^{(v)}(x)\right\|_{B\left(G_{n}\right)} \leq C(n+1)^{v}, \quad v=0,1, \quad x \in[0, \pi], \\
\left\|\psi_{n}(x)-\tilde{\psi}_{n}(x)\right\|_{B\left(G_{n}\right)} \leq C \Omega \eta_{n}, \quad\left\|\psi_{n}^{\prime}(x)-\tilde{\psi}_{n}^{\prime}(x)\right\|_{B\left(G_{n}\right)} \leq C \Omega, \quad x \in[0, \pi] .
\end{gathered}
$$

where

$$
\eta_{n}:=\left(\sum_{k=0}^{\infty} \frac{1}{(k+1)^{2}(|n-k|+1)^{2}}\right)^{1 / 2} .
$$

By virtue of Lemma 20, the matrix-functions $\varphi_{n q i}(x):=\psi_{n}\left(x, \rho_{n q i}\right)$ satisfy the following estimates

$$
\begin{gathered}
\left\|\varphi_{n q i}^{(v)}(x)\right\| \leq C(n+1)^{v}, \quad v=0,1, \\
\left\|\varphi_{n q i}(x)-\tilde{\varphi}_{n q i}(x)\right\| \leq C \Omega \eta_{n}, \quad\left\|\varphi_{n q i}^{\prime}(x)-\tilde{\varphi}_{n q i}^{\prime}(x)\right\| \leq C \Omega, \quad q=\overline{1, m}, \\
\left\|\varphi_{n q i}(x)-\varphi_{n l j}(x)\right\| \leq C\left|\rho_{n q i}-\rho_{n l j}\right|, \quad q, l=\overline{1, m}, i, j=0,1 .
\end{gathered}
$$


Further, we construct the matrix-functions $\varphi(x, \lambda)$ and $\Phi(x, \lambda)$ by the formulas

$$
\begin{aligned}
& \varphi(x, \lambda)=\tilde{\varphi}(x, \lambda)-\sum_{k=0}^{\infty} \sum_{l=1}^{m} \sum_{j=0}^{1}(-1)^{j} \varphi_{k l j}(x) \alpha_{k l j}^{\prime} \frac{\left\langle\tilde{\varphi}_{k l j}^{*}(x), \tilde{\varphi}(x, \lambda)\right\rangle}{\lambda-\lambda_{k l j}}, \\
& \Phi(x, \lambda)=\tilde{\Phi}(x, \lambda)-\sum_{k=0}^{\infty} \sum_{l=1}^{m} \sum_{j=0}^{1}(-1)^{j} \varphi_{k l j}(x) \alpha_{k l j}^{\prime} \frac{\left\langle\tilde{\varphi}_{k l j}^{*}(x), \tilde{\Phi}(x, \lambda)\right\rangle}{\lambda-\lambda_{k l j}},
\end{aligned}
$$

(see (30), (31)) and the boundary value problem $L(Q(x), h, H)$ via (47). Clearly, $\varphi\left(x, \lambda_{n q i}\right)=$ $\varphi_{n q i}(x)$.

Using estimates (54), one can show that the entries of $\varepsilon_{0}(x)$ are absolutely continuous and the entries of $\varepsilon(x)$ belong to $L_{2}(0, \pi)$. Consequently, we get

Lemma 21. $Q(x) \in L_{2}\left((0, \pi), \mathbb{C}^{m \times m}\right)$.

Lemma 22. The following relations hold

$$
\begin{gathered}
\ell \varphi_{n q i}(x)=\lambda_{n q i} \varphi_{n q i}(x), \quad \ell \varphi(x, \lambda)=\lambda \varphi(x, \lambda), \quad \ell \Phi(x, \lambda)=\lambda \Phi(x, \lambda), \\
\varphi(0, \lambda)=I_{m}, \quad \varphi^{\prime}(0, \lambda)=h, \quad U(\Phi)=I_{m}, \quad V(\Phi)=0_{m} .
\end{gathered}
$$

Proof. Let us prove only the relation $V(\Phi)=0_{m}$, since other ones can be obtained similarly to the scalar case [4]. It follows from (47) and (55), that

$$
\begin{aligned}
\tilde{V}(\tilde{\varphi})= & V(\varphi)+\sum_{k=0}^{\infty} \sum_{l=1}^{m}\left(V\left(\varphi_{k l 0}\right) \alpha_{k l 0}^{\prime} \tilde{D}\left(\pi, \lambda, \lambda_{k l 0}\right)-V\left(\varphi_{k l 1}\right) \alpha_{k l 1}^{\prime} \tilde{D}\left(\pi, \lambda, \lambda_{k l 1}\right)\right), \\
\tilde{V}\left(\tilde{\varphi}_{n q 1}\right) \alpha_{n q 1}= & V\left(\varphi_{n q 1}\right) \alpha_{n q 1}+\sum_{k=0}^{\infty} \sum_{l=1}^{m}\left(V\left(\varphi_{k l 0}\right) \alpha_{k l 0}^{\prime} \tilde{D}\left(\pi, \lambda_{n q 1}, \lambda_{k l 0}\right) \alpha_{n q 1}\right. \\
& \left.-V\left(\varphi_{k l 1}\right) \alpha_{k l 1}^{\prime} \tilde{D}\left(\pi, \lambda_{n q 1}, \lambda_{k l 1}\right) \alpha_{n q 1}\right) .
\end{aligned}
$$

Using (18) and (51), we derive

$$
\sum_{k=0}^{\infty} \sum_{l=1}^{m} V\left(\varphi_{k l 0}\right) \alpha_{k l 0}^{\prime} \tilde{D}\left(\pi, \lambda_{n q 1}, \lambda_{k l 0}\right) \alpha_{n q 1}=0 .
$$

Taking (27) into account, we obtain

$$
\begin{gathered}
\int_{0}^{\pi} f(x) \tilde{\varphi}_{n q 1}(x) \alpha_{n q 1} d x=0_{m}, \quad n \geq 0, q=\overline{1, m}, \\
f(x):=\sum_{k=0}^{\infty} \sum_{l=1}^{m} V\left(\varphi_{k l 0}\right) \alpha_{k l 0}^{\prime} \tilde{\varphi}_{k l 0}^{*}(x),
\end{gathered}
$$

Let us prove that $f \in L_{2}\left((0, \pi), \mathbb{C}^{m \times m}\right)$. Indeed, $\varphi(x, \lambda)$ is a solution of equation (1), therefore the relation (19) holds and

$$
V\left(\varphi_{k l 0}\right)=(-1)^{k}\left(\omega-\omega_{l} I_{m}\right)+K_{k} .
$$


By virtue of (8), $\left\{\left\|V\left(\varphi_{k l 0}\right) \alpha_{k l 0}^{\prime}\right\|\right\} \in l_{2}$. Similarly to asymptotic relation (21), we get $\tilde{\varphi}_{k l 0}^{*}(x)=$ $\cos k x I_{m}+O\left(k^{-1}\right)$, and the series in (57) converges in $L_{2}$. Hence $f(x)$ belongs to $L_{2}$.

The system of linearly independent columns of the matrices $\varphi_{k l 1}(x) \alpha_{k l 1}$ is complete in $L_{2}\left((0, \pi), \mathbb{C}^{m}\right)$ by Lemma 17 . Hence, it follows from (57), that $f(x) \equiv 0$,

By Lemma 19, linearly independent rows of the matrices $\alpha_{k l 0}^{\prime} \varphi_{k l 0}^{*}(x)$ form a basis in $L_{2}\left((0, \pi), \mathbb{C}^{m, T}\right)$. Therefore, the terms in (58) can not differ from zero. Hence

$$
V\left(\varphi_{k l 0}\right) \alpha_{k l 0}^{\prime}=0_{m}, \quad k \geq 0, \quad l=\overline{1, m}
$$

Using (47) and (56), we obtain

$$
\tilde{V}(\tilde{\Phi})=V(\Phi)+\sum_{k=0}^{\infty} \sum_{l=1}^{m}\left(V\left(\varphi_{k l 0}\right) \alpha_{k l 0}^{\prime} \frac{\left\langle\tilde{\varphi}_{k l 0}^{*}(x), \tilde{\Phi}(x, \lambda)\right\rangle_{x=\pi}}{\lambda-\lambda_{k l 0}}-V\left(\varphi_{k l 1}\right) \alpha_{k l 1}^{\prime} \frac{\left\langle\tilde{\varphi}_{k l 1}^{*}(x), \tilde{\Phi}(x, \lambda)\right\rangle_{x=\pi}}{\lambda-\lambda_{k l 1}}\right)
$$

Applying (59) and the following relations:

$$
\tilde{V}(\tilde{\Phi})=0_{m}, \quad \alpha_{k l 1} \frac{\left\langle\tilde{\varphi}_{k l 1}^{*}(x), \tilde{\Phi}(x, \lambda)\right\rangle_{x=\pi}}{\lambda-\lambda_{k l 1}}=\alpha_{k l 1} \frac{\tilde{V}^{*}\left(\tilde{\varphi}_{k l 1}^{*}\right) \tilde{\Phi}(\pi, \lambda)-\tilde{\varphi}_{k l 1}^{*}(\pi) \tilde{V}(\tilde{\Phi})}{\lambda-\lambda_{k l 1}}=0_{m},
$$

we conclude that $V(\Phi)=0_{m}$.

In order to finish the proof of Theorem 1, it remains to show that the constructed boundary value problem $L(Q, h, H)$ belongs to $A_{1,2}(\omega)$ and the given data $\left\{\lambda_{n q}, \alpha_{n q}\right\}$ coincide with the spectral data of $L$. In view of Lemma 22, the matrix-function $\Phi(x, \lambda)$ is the Weyl solution of $L$. Let us get the representation for the Weyl matrix:

$$
\begin{aligned}
M(\lambda)=\Phi(0, \lambda)= & \tilde{M}(\lambda)-\sum_{k=0}^{\infty} \sum_{l=1}^{m} \sum_{j=0}^{1}(-1)^{j} \varphi_{k l j}(0) \alpha_{k l j}^{\prime} \frac{\left\langle\tilde{\varphi}_{k l j}^{*}(x), \tilde{\Phi}(x, \lambda)\right\rangle_{x=0}}{\lambda-\lambda_{k l j}} \tilde{M}(\lambda) \\
& +\sum_{k=0}^{\infty} \sum_{l=1}^{m}\left(\frac{\alpha_{k l 0}^{\prime}}{\lambda-\lambda_{k l 1}}-\frac{\alpha_{k l 1}^{\prime}}{\lambda-\lambda_{k l 1}}\right) .
\end{aligned}
$$

Using the equality (see [9])

$$
\tilde{M}(\lambda)=\sum_{k=0}^{\infty} \sum_{l=1}^{m} \frac{\alpha_{k l 1}^{\prime}}{\lambda-\lambda_{k l 1}}
$$

we arrive at

$$
M(\lambda)=\sum_{k=0}^{\infty} \sum_{l=1}^{m} \frac{\alpha_{k l 0}^{\prime}}{\lambda-\lambda_{k l 0}}
$$

Consequently, $\left\{\lambda_{k l 0}\right\}$ are simple poles of the Weyl matrix $M(\lambda)$, and $\left\{\alpha_{k l 0}\right\}$ are residues at the poles. So $L \in A_{1,2}(\omega)$, and $\Lambda$ is the spectral data of $L$. Theorem 1 is proved. 


\section{The self-adjoint case}

Suppose $Q(x)=Q^{\dagger}(x)$ a.e. on $[0, \pi], h=h^{\dagger}, H=H^{\dagger}$. In this case, the matrix $\omega=h+H+$ $\frac{1}{2} \int_{0}^{\pi} Q(x) d x$ is Hermitian. Therefore, one can diagonalize it, applying a unitary transform. Without loss of generality, we consider $L \in A(\omega), \omega=\omega^{\dagger} \in \mathscr{D}$.

The eigenvalues $\lambda_{n q}$ are real, and the poles of the matrix function $\left(V(\varphi(x, \lambda))^{-1}\right.$ are simple [11]. Furthermore, it is easy to check that $\varphi^{*}(x, \lambda)=\varphi^{\dagger}(x, \bar{\lambda}), S^{*}(x, \lambda)=S^{\dagger}(x, \bar{\lambda})$. Consequently, $M^{*}(\lambda)=M^{\dagger}(\bar{\lambda})=M(\lambda)$ and $\alpha_{n q}=\alpha_{n q}^{\dagger}$, for all $n \geq 0, q=\overline{1, m}$. By Lemma 7 ,

$$
\alpha_{n q}=\alpha_{n q}^{\dagger} \int_{0}^{\pi} \varphi^{\dagger}\left(x, \lambda_{n q}\right) \varphi\left(x, \lambda_{n q}\right) d x \alpha_{n q} \geq 0 .
$$

Taking the last fact together with asymptotics (7), we conclude, that $\left\|\alpha_{n q}\right\| \leq C, n \geq 0, q=\overline{1, m}$.

Thus, we have shown that Assumptions 1 and 2 hold automatically in the self-adjoint case. Moreover, we have proved (S) in Theorem 2. Note that (E) by necessity was proved in Lemma 8. So we have finished the proof of the necessity.

In order to prove the sufficiency in Theorem 2, it remains to show that the solvability of the main equation (34) follows from (E) together with other conditions.

Let data $\left\{\lambda_{n q}, \alpha_{n q}\right\}_{n \geq 0, q=\overline{1, m}} \in \mathrm{Sp}$, satisfying the conditions of Theorem 2, be given. Choose a model problem $\tilde{L} \in A(\omega)$, construct $\tilde{\psi}(x), \tilde{R}(x)$, and consider the main equation (34).

Lemma 23. For each fixed $x \in[0, \pi]$, the operator $I+\tilde{R}(x)$, acting from $B$ to $B$, has a bounded inverse operator, and the main equation (34) has a unique solution $\psi(x) \in B$.

Proof. By Lemma 12 the operator $\tilde{R}(x)$ is compact. Therefore it is sufficient to prove that the homogeneous equation

$$
\gamma(x)(I+\tilde{R}(x))=0
$$

where $\gamma(x) \in B$, has only the zero solution. Let $\gamma(x)=\left\{\gamma_{n}(x)\right\}_{n \geq 0} \in B$ be a solution of (60). Denote $\gamma_{n q i}(x)=\gamma_{n}\left(x, \rho_{n q i}\right)$. Then

$$
\gamma_{n q i}(x)+\sum_{k=0}^{\infty} \sum_{l=1}^{m}\left(\gamma_{k l 0}(x) \alpha_{k l 0}^{\prime} \tilde{D}\left(x, \lambda_{n q i}, \lambda_{k l 0}\right)-\gamma_{k l 1}(x) \alpha_{k l 1}^{\prime} \tilde{D}\left(x, \lambda_{n q i}, \lambda_{k l 1}\right)\right)=0_{m},
$$

and the following estimates are valid

$$
\left\|\gamma_{n q i}(x)\right\| \leq C, \quad\left\|\gamma_{n q i}(x)-\gamma_{n l j}(x)\right\| \leq C\left|\rho_{n q i}-\rho_{n l j}\right|, \quad n \geq 0, q, l=\overline{1, m}, i, j=0,1
$$

Construct the matrix-functions $\gamma(x, \lambda), \Gamma(x, \lambda)$ and $\mathscr{B}(x, \lambda)$ by the formulas

$$
\gamma(x, \lambda)=-\sum_{k=0}^{\infty} \sum_{l=1}^{m}\left(\gamma_{k l 0}(x) \alpha_{k l 0}^{\prime} \tilde{D}\left(x, \lambda, \lambda_{k l 0}\right)-\gamma_{k l 1}(x) \alpha_{k l 1}^{\prime} \tilde{D}\left(x, \lambda, \lambda_{k l 1}\right)\right),
$$




$$
\begin{gathered}
\Gamma(x, \lambda)=-\sum_{k=0}^{\infty} \sum_{l=1}^{m}\left[\gamma_{k l 0}(x) \alpha_{k l 0}^{\prime} \frac{\left\langle\tilde{\varphi}_{k l 0}^{*}(x), \tilde{\Phi}(x, \lambda)\right\rangle}{\lambda-\lambda_{k l 0}}-\gamma_{k l 1}(x) \alpha_{k l 1}^{\prime} \frac{\left\langle\tilde{\varphi}_{k l 1}^{*}(x), \tilde{\Phi}(x, \lambda)\right\rangle}{\lambda-\lambda_{k l 1}}\right], \\
\mathscr{B}(x, \lambda)=\Gamma(x, \lambda) \gamma^{\dagger}(x, \bar{\lambda}) .
\end{gathered}
$$

In view of (27), the matrix-function $\gamma(x, \lambda)$ is entire in $\lambda$ for each fixed $x$. The functions $\Gamma(x, \lambda)$ and $\mathscr{B}(x, \lambda)$ are meromorphic in $\lambda$ with simple poles $\lambda_{n q i}$. According to $(61), \gamma\left(x, \lambda_{n q i}\right)=$ $\gamma_{n q i}(x)$. We calculate residues of $\mathscr{B}(x, \lambda)$ (for simplicity we assume that $\left.\left\{\lambda_{n q 0}\right\} \cap\left\{\lambda_{n q 1}\right\}=\varnothing\right)$ :

$$
\operatorname{Res}_{\lambda=\lambda_{n q 0}} \mathscr{B}(x, \lambda)=\gamma\left(x, \lambda_{n q 0}\right) \alpha_{n q 0} \gamma^{\dagger}\left(x, \lambda_{n q 0}\right), \quad \operatorname{Res}_{\lambda=\lambda_{n q 1}} \mathscr{B}(x, \lambda)=0_{m}
$$

Consider the integral

$$
I_{N}(x)=\frac{1}{2 \pi i} \int_{\Gamma_{N}} \mathscr{B}(x, \lambda) d \lambda,
$$

where $\Gamma_{N}=\left\{\lambda:|\lambda|=(N+1 / 2)^{2}\right\}$. Let us show that for each fixed $x \in[0, \pi]$

$$
\lim _{N \rightarrow \infty} I_{N}(x)=0_{m}
$$

Indeed, transforming (63) similarly to (50), and using (28), (62) and the estimates

$$
\begin{array}{r}
\left\|\tilde{D}\left(x, \lambda, \lambda_{k l j}\right)\right\| \leq \frac{C \exp (|\tau| x)}{|\rho-k|+1}, \quad \| \tilde{D}\left(x, \lambda, \lambda_{k l j}\right)-\tilde{D}\left(x, \lambda, \lambda_{k q i} \| \leq \frac{C\left|\rho_{k l j}-\rho_{k q i}\right| \exp (|\tau| x)}{|\rho-k|+1},\right. \\
k \geq 0, l, q=\overline{1, m}, i, j=0,1,
\end{array}
$$

we get

$$
\|\gamma(x, \lambda)\| \leq C(x) \exp (|\tau| x) \sum_{k=0}^{\infty} \frac{\xi_{k}}{|\rho-k|+1}, \quad \operatorname{Re} \rho \geq 0 .
$$

Similarly, using (64) we obtain for sufficiently large $\rho^{*}>0$ :

$$
\|\Gamma(x, \lambda)\| \leq \frac{C(x)}{|p|} \exp (-|\tau| x) \sum_{k=0}^{\infty} \frac{\xi_{k}}{|\rho-k|+1}, \operatorname{Re} \rho \geq 0,|\rho| \geq \rho^{*},|\rho-k|>\delta>0 .
$$

Then

$$
\|\mathscr{B}(x, \lambda)\| \leq \frac{C(x)}{|\rho|}\left(\sum_{k=0}^{\infty} \frac{\xi_{k}}{|\rho-k|+1}\right)^{2} \leq \frac{C(x)}{|\rho|^{3}}, \quad \lambda \in \Gamma_{N} .
$$

This estimate yields $\lim _{N \rightarrow \infty} I_{N}(x)=0_{m}$.

On the other hand, calculating the integral $I_{N}(x)$ by the residue theorem, we arrive at

$$
\sum_{k=0}^{\infty} \sum_{l=1}^{m} \gamma_{k l 0}(x) \alpha_{k l 0}^{\prime} \gamma_{k l 0}^{\dagger}(x)=0_{m}
$$

Since $\alpha_{k l 0}=\alpha_{k l 0}^{\dagger} \geq 0$, we get

$$
\gamma_{k l 0}(x) \alpha_{k l 0} \gamma_{k l 0}^{\dagger}(x)=0_{m}
$$




$$
\gamma\left(x, \lambda_{k l 0}\right) \alpha_{k l 0}=0_{m}, \quad k \geq 0, \quad l=\overline{1, m} .
$$

Since $\gamma(x, \lambda)$ is entire in $\lambda$, and

$$
\gamma(x, \lambda)=O(\exp (|\tau| x))
$$

for each fixed $x \in[0, \pi]$, according (E), we get $\gamma(x, \lambda) \equiv 0_{m}$. Therefore $\gamma_{n q i}(x)=0_{m}$ for all $n \geq 0$, $q=\overline{1, m}, i=0,1$, i. e. the homogeneous equation (60) has only the zero solution.

Thus, the proof of Theorem 2 is finished. Some discussion on condition (E) with examples is provided in [11]. We can also give an alternative formulation of necessary and sufficient conditions with condition (C) instead of (E).

Theorem 5. Let $\omega=\omega^{\dagger} \in \mathscr{D}$. For data $\left\{\lambda_{n q}, \alpha_{n q}\right\}_{n \geq 0, q=\overline{1, m}} \in S p$ to be the spectral data for a certain self-adjoint problem $L \in A(\omega)$ it is necessary and sufficient to satisfy the following conditions.

(A) The asymptotics (3), (7), (8) (9) are valid.

(R) The ranks of the matrices $\alpha_{n q}$ coincide with the multiplicities of the corresponding values $\lambda_{n q}$.

(S) All $\lambda_{n q}$ are real, $\alpha_{n q}=\left(\alpha_{n q}\right)^{\dagger}, \alpha_{n q} \geq 0$ for all $n \geq 0, q=\overline{1, m}$.

(C) The system of functions

$$
\cos \rho_{n q} x \mathscr{E}_{n q}^{(i)}, \quad n \geq 0, q=\overline{1, m}, i=\overline{1, m_{n q}},
$$

is complete in $L_{2}\left((0, \pi), \mathbb{C}^{m}\right)$.

Condition (C) was used by Ya.V. Mykytyuk and N.S. Trush [12] in the characterization of the spectral data for the self-adjoint matrix Sturm-Liouville operator with the potential from $W_{2}^{-1}$. In spite of the fact, that this class is wider than our class $L_{2}$, these are two parallel results for different classes. Moreover, for $W_{2}^{-1}$ the asymptotics of eigenvalues and weight matrices are more rough, that makes the class $W_{2}^{-1}$ easier for investigation. It does not require our technique with complicated division of eigenvalues into groups. Now we have shown, that condition (C) can be used in our case with our method, so there is no principal difference with the work [12] in this particular point.

It was established in Section 5, that $(\mathrm{C})$ is weaker than $(\mathrm{E})$, but $(\mathrm{C})$ is equivalent to $(\mathrm{PW})$. One can easily show that everywhere in our proofs (namely, in Lemmas 17 and 23), we apply this type of conditions to functions $\gamma(\lambda) \in$ PW. Thus, both Theorems 2 and 5 are valid.

\section{Acknowledgement}

This work was supported by Grant 1.1660.2017/4.6 of the Russian Ministry of Education and Science. 


\section{References}

[1] V. A. Marchenko, Sturm-Liouville Operators and their Applications, Naukova Dumka, Kiev (1977) (Russian); English transl., Birkhauser, 1986.

[2] B. M. Levitan, Inverse Sturm-Liouville Problems, Nauka, Moscow (1984) (Russian); English transl., VNU Sci. Press, Utrecht, 1987.

[3] J. Pöschel and E. Trubowitz, Inverse Spectral Theory, New York, Academic Press, 1987.

[4] G. Freiling and V. Yurko, Inverse Sturm-Liouville problems and their applications, Huntington, NY: Nova Science Publishers, 305 p., 2001.

[5] Z. S. Agranovich and V. A. Marchenko, The inverse problem of scattering theory [in Russian], KSU, Kharkov, 1960; Gordon and Breach, New York, 1963 (Eng. Transl.).

[6] R. Carlson, An inverse problem for the matrix Schrödinger equation, J. Math. Anal. Appl., 267 (2002), 564575.

[7] M. M. Malamud, Uniqueness of the matrix Sturm-Liouville equation given a part of the monodromy matrix, and Borg type results, Sturm-Liouville Theory, Birkhäuser, Basel, (2005), 237-270.

[8] V. A. Yurko, Inverse problems for matrix Sturm-Liouville operators, Russian J. Math. Phys., 13, no.1 (2006), $111-118$.

[9] V. A. Yurko, Inverse problems for the matrix Sturm-Liouville equation on a finite interval, Inverse Problems, 22 (2006), 1139-1149.

[10] D. Chelkak and E. Korotyaev, Weyl-Titchmarsh functions of vector-valued Sturm-Liouville operators on the unit interval, J. Func. Anal. 257 (2009), 1546-1588.

[11] N. Bondarenko, Spectral analysis for the matrix Sturm-Liouville operator on a finite interval, Tamkang J. Math., 42: 3 (2011), 305-327.

[12] Ya. V. Mykytyuk and N. S. Trush, Inverse spectral problems for Sturm-Liouville operators with matrix-valued potentials, Inverse Problems, 26 (2010), 015009.

[13] N. Bondarenko, An inverse spectral problem for the matrix Sturm-Liouville operator on the half-line, Boundary Value Problems. 2015:15.

[14] N. Bondarenko, Inverse scattering on the line for the matrix Sturm-Liouville equation, J. Differential Equations, 262 (2017), Issue 3, 2073-2105.

[15] V. A. Yurko, An inverse spectral problem for singular non-self-adjoint differential systems, Sbornik: Mathematics (2004) 195:12, 1823-1854.

[16] V. A. Yurko, Reconstruction of non-self-adjoint differential systems on the half-Line from the Weyl matrix, Mathematical Notes (2004), 76, no. 2, 296-302.

[17] V. A. Yurko, An inverse problem for differential systems on a finite interval in the case of multiple roots of the characteristic polynomial, Diff. Equ., 41 (2005), no. 6, 818-823.

[18] V. A. Yurko, Method of Spectral Mappings in the Inverse Problem Theory. Inverse and Ill-Posed Problems Series, Utrecht: VSP, 2002.

[19] S. A. Buterin, On inverse spectral problem for non-selfadjoint Sturm-Liouville operator on a finite interval, Journal of Mathematical Analysis and Applications, 335 (2007), Issue 1, 739-749.

[20] S. A. Buterin, C.-T. Shieh, and V. A. Yurko, Inverse spectral problems for non-selfadjoint second-order differential operators with Dirichlet boundary conditions, Boundary Value Problems (2013), 2013:180, 1-24.

[21] M. A. Naimark, Linear Differential Operators, 2nd ed., Nauka, Moscow (1969); English transl. of 1st ed., Parts I,II, Ungar, New York $(1967,1968)$.

Chair of Applied Mathematics and Physics, Samara National Research University, 34, Moskovskoye Shosse, Samara 443086, Russia.

Department of Mechanics and Mathematics, Saratov State University, Astrakhanskaya 83, Saratov 410012, Russia

E-mail: BondarenkoNP@info.sgu.ru 